\title{
Feasible Joint Posterior Beliefs
}

\section{Itai Arieli}

Technion-Israel Institute of Technology

\section{Yakov Babichenko}

Technion-Israel Institute of Technology

\section{Fedor Sandomirskiy}

Technion - Israel Institute of Technology and Higher School of Economics, St. Petersburg

\section{Omer Tamuz}

California Institute of Technology

\begin{abstract}
We study the set of possible joint posterior belief distributions of a group of agents who share a common prior regarding a binary state and who observe some information structure. For two agents, we introduce a quantitative version of Aumann's agreement theorem and show that it is equivalent to a characterization of feasible distributions from a 1995 work by Dawid and colleagues. For any number of agents, we characterize feasible distributions in terms of a "no-trade" condition. We use these characterizations to study information structures with independent posteriors. We also study persuasion problems with multiple receivers, exploring the extreme feasible distributions.
\end{abstract}

This paper greatly benefited from multiple suggestions and comments of our colleagues. We are grateful to (in alphabetic order) Kim Border, Ben Brooks, Laura Doval, Piotr Dworczak, Nikita Gladkov, Sergiu Hart, Kevin He, Aviad Heifetz, Yuval Heller, Matthew Jackson, Eliott Lipnowski, Jeffrey Mensch, Benny Moldovanu, Inés Moreno de Barreda, Stephen Morris, Alexander Nesterov, Abraham Neyman, Michael Ostrovsky, Thomas Palfrey, Jim Pitman, Luciano Pomatto, Doron Ravid, Marco Scarsini, Eilon Solan, Theodore Zhu, Gabriel

Electronically published July 7, 2021

[ Journal of Political Economy, 2021, vol. 129, no. 9]

(C) 2021 by The University of Chicago. All rights reserved. 0022-3808/2021/12909-0003\$10.00 


\section{Introduction}

The question of whether agents' observed behavior is compatible with rationality is fundamental and pervasive in microeconomics. A particularly interesting case is that of beliefs: when are agents' beliefs compatible with Bayes's law?

Consider a single agent who has a prior belief regarding an uncertain event and updates to a posterior belief after receiving some information. An analyst observes the prior and the distribution of posteriors but not the information structure. When is the distribution of posterior beliefs feasible? That is, when is it compatible with Bayesian updating according to some information structure? Feasibility in this case corresponds to what is known as the martingale condition: the expectation of the posterior must equal the prior. This fundamental result is known as the "splitting lemma" (see Blackwell 1951; Aumann and Maschler 1995), and it is a key tool in the theories of Bayesian persuasion (Kamenica and Gentzkow 2011) and of games with incomplete information (Aumann and Maschler 1995).

Thus, in the case of a single agent, the analyst can readily determine feasibility. ${ }^{1}$ We ask the same question, but regarding groups of more than one agent. In particular, we consider a group of agents for which one can observe a common prior regarding a binary state as well as a joint distribution of posteriors. This distribution is said to be feasible if it is the result of Bayesian updating induced by some joint information structure observed by the agents. Is there also in this case a simple characterization of feasibility that does not require the analyst to test infinitely many possible information structures?

Clearly, feasibility implies that each agent's posterior distribution must satisfy the martingale condition. An additional important obstruction to feasibility is given by Aumann's seminal agreement theorem (Aumann 1976). Aumann showed that rational agents cannot agree to disagree on posteriors: when posteriors are common knowledge, they cannot be unequal.

\footnotetext{
Ziegler, and seminar participants at Bar-Ilan University, Caltech, Hebrew University, the Higher School of Economics St. Petersburg, Technion, Tel Aviv University, Stanford, and the University of California San Diego. Arieli is supported by the Ministry of Science and Technology (2028255). Babichenko is supported by a BSF (United States-Israel Binational Science Foundation) award (2018397). Sandomirskiy is supported by the Lady Davis Foundation, by grant 19-01-00762 of the Russian Foundation for Basic Research, by the European Research Council (ERC) under the European Union's Horizon 2020 research and innovation program (740435), and by the Basic Research Program of the National Research University Higher School of Economics. Tamuz is supported by a grant from the Simons Foundation (419427), by a BSF award (2018397), and by a Sloan Fellowship.

1 The question becomes more subtle when the agent is exposed to a collection of information sources. Brooks, Frankel, and Kamenica (2019) demonstrated that the feasible family of distributions that arises as we vary the subset of information sources does not admit a simple characterization even in the single-agent case.
} 
This implies that feasibility is precluded if, for example, agent 1 has posterior $1 / 5$ whenever agent 2 has posterior $2 / 5$ and likewise agent 2 has posterior $2 / 5$ whenever agent 1 has posterior $1 / 5$. There are, however, examples of distributions that are not feasible even though they do not involve agents who agree to disagree as above. Thus, the agreement theorem does not provide a necessary and sufficient condition for feasibility. Our first result is a quantitative version of the agreement theorem: a relaxation that constrains beliefs to be approximately equal when they are approximately common knowledge.

For the case of two agents, a characterization of feasible distributions was obtained by Dawid et al. (1995). Their criterion of feasibility takes the form of a family of inequalities, which arise from results due to Kellerer (1961) and Strassen (1965). As we explain, these inequalities correspond exactly to those in our quantitative agreement theorem, which thus provides a necessary and sufficient condition for feasibility: a joint posterior belief distribution is feasible if and only if agents do not approximately agree to disagree.

For any number of agents, we find a characterization of feasibility via a "no-trade" condition. To derive this condition, we introduce - as an analytic tool - a mediator who trades a good of uncertain value with the agents. When an agent trades, it is at a price that reflects her expected value, and thus the agents do not gain or lose in expectation. This implies that the mediator likewise does not turn a profit, and so by bounding the mediator's profit by zero we attain an obviously necessary condition for feasibility. We show that this condition is also sufficient. Thus, for any number of agents, a joint posterior belief distribution is feasible if and only if no mediator can extract an expected profit from a trading scheme in which each agent expects to lose nothing.

We apply these characterizations to study independent joint posterior belief distributions: these are induced by information structures in which each agent receives nontrivial information regarding the state and yet gains no information about the others' posterior. We give a simple condition for feasibility of independent distributions in the case of two agents with identically distributed, symmetric posteriors: such distributions are feasible if and only if the uniform distribution on $[0,1]$ is a mean-preserving spread of the single-agent belief distribution.

The set of feasible distributions plays an important role in Bayesian persuasion problems, in particular in what we call "first-order" Bayesian persuasion. A first-order Bayesian persuasion problem includes a single sender and multiple receivers. First, the sender chooses an information structure to be revealed to the agents. Then, each receiver chooses an action. Finally, both the receivers and the sender receive payoffs. The sender's utility depends generally on the receivers' actions. The key assumption is that each receiver's utility depends only on the unknown state 
and her own action, and so only her first-order beliefs matter for her choice; we therefore refer to these problems as first-order persuasion problems. As each receiver's action depends only on her own posterior, and as the sender's utility depends on the actions of the receivers, the sender's problem is to choose an optimal feasible joint posterior belief distribution for the receivers.

As an example of a first-order Bayesian persuasion problem, we study the problem of a "polarizing" sender who wishes to maximize the difference between two receivers' posteriors. This example highlights the limitations imposed by feasibility, as the sender cannot hope to always achieve complete polarization in which one receiver has posterior 0 and the other posterior 1; such joint posterior distributions are precluded by the agreement theorem. We solve this problem in some particular regimes and show that nontrivial solutions exist in others, depending on the details of how the difference between posteriors is quantified.

A related question is how anticorrelated feasible posteriors can be. While they can clearly be perfectly correlated, by the agreement theorem they cannot be perfectly anticorrelated. This question can be phrased as a first-order Bayesian persuasion problem, which we solve. When the two states are a priori equally likely, we show that the covariance between posteriors has to be at least $-1 / 32$ and construct a feasible distribution, supported on four points, that achieves this bound. ${ }^{2}$

The question of first-order Bayesian persuasion is closely tied to the study of the extreme points of the set of feasible distributions, as the sender's optimum is always achieved at an extreme point. In the single-agent case, the well-known concavification argument of Aumann and Maschler (1995) and Kamenica and Gentzkow (2011) shows that every extreme point of the set of feasible posterior distributions has support of size at most two. In contrast, we show that for two or more agents there exist extreme points with countably infinite support. In the other direction, we show that every extreme point is supported on a set that is small, in the sense of having a Lebesgue measure of zero. To this end, we do not use our characterization of feasibility but rather use a classical result of Lindenstrauss (1965) regarding extreme points of the set of measures with given marginals. Likewise, our analysis of first-order persuasion is not based on the aforementioned characterizations of feasibility; to study these problems, we exploit the fact that conditional expectations are orthogonal projections in the Hilbert space of square-integrable random variables.

This work leaves open a number of interesting questions. In particular, we leave for future studies the extension of our work beyond the setting of a binary state and common priors. Likewise, the study of the extreme

${ }^{2}$ The questions of first-order persuasion for polarization and anticorrelation were mentioned by Burdzy and Pitman (2020, problem 5.2) as open problems. 
feasible distributions is far from complete. For example, we do not have a simple characterization of extreme points. We also do not know whether there are nonatomic extreme points.

Related literature: coherent experts' opinions.-The study of feasible joint distributions of posteriors was pioneered by Dawid et al. (1995). They were motivated by the question of aggregating the opinions of experts who rely on different information sources. As a by-product of their analysis, Dawid et al. (1995) characterized the set of feasible distributions for two agents and a binary state; the detailed discussion of this result is in section II. Their foundational paper and the mathematical literature inspired by it seem to be known little by the economic theory community. ${ }^{3}$ We refer to Burdzy and Pal (2019) and Burdzy and Pitman (2020) for the references to the literature on experts, summary of the results known in the two-agent case (the main focus of this literature), and tight bounds on the probability that the pair of posteriors differ by more than $\delta$. Maximizing the latter probability can be seen as an example of a first-order Bayesian persuasion problem. Another example is offered by Dubins and Pitman (1980), who found the distribution maximizing the expected maximal posterior for any number of agents. A particular case of this result for two agents follows from our analysis; see the discussion after proposition 5. Independently and concurrently, Cichomski (2020) proved an analog of our proposition 5, using a Hilbert-space technique similar to ours. Gutmann et al. (1991) discovered an analog of the characterization by Dawid et al. (1995) in the particular case of independent posteriors and demonstrated that the uniform distribution on the square is feasible; they did not discover the special role played by this distribution (see our proposition 2).

Related literature: information design and necessary conditions for feasibility.Recently, several necessary conditions for feasibility appeared in the economic literature studying information design with bounded rational receivers. Independently from us, Ziegler (2020) considered an information design setting with two receivers and derived a necessary condition for feasibility. His condition is sufficient for the case of binary signals. We further discuss Ziegler's condition in appendix D. Another necessary condition was found by Levy, Moreno de Barreda, and Razin (2020, their proposition 4); it is equivalent to the corollary of Aumann's agreement theorem (corollary A) and hence is not sufficient. Levy, Moreno de Barreda, and Razin (2020) also offered several recipes for how to construct feasible distributions starting from infeasible ones.

Related literature: alternative approaches to multiagent information design.Mathevet, Perego, and Taneva (2020) studied Bayesian persuasion with multiple receivers and a finite number of signals. They found an implicit

\footnotetext{
${ }^{3}$ We are indebted to Jim Pitman for introducing us to this literature.
} 
characterization of feasibility: considering the entire belief hierarchy, they showed that feasibility is equivalent to the consistency of the hierarchy. ${ }^{4}$ Arieli and Babichenko (2019), Bergemann and Morris (2019), and Taneva (2019) related the optimal information disclosure to the best Bayes-correlated equilibrium from the sender's perspective. Even if receivers' actions are not free of externalities, finding the best such equilibrium leads to a linear program. This linear program happens to be tractable for a finite number of actions (Arieli and Babichenko 2019 and Taneva 2019 focused on the binary case). Our approach is conceptually closer to the geometric point of view on persuasion of Kamenica and Gentzkow (2011), where the distribution of posteriors plays the key role. Our approach helps "visualize" the solution and does not require the set of actions to be finite ${ }^{5}$ however, it is limited to the firstorder persuasion problems, that is, we rule out strategic externalities. ${ }^{6}$

Related literature: common prior and no-trade.-A question related to ours is the common-prior problem studied in the literature on interactive epistemology. Concretely, an Aumann knowledge-partition model with finite state space and agents is considered. Each agent has a partition over the state space, and to each partition element corresponds a posterior probability that is supported on that partition element. The question is whether there exists a common prior. a single probability distribution over the state space that gives rise to all the posterior distributions by means of conditional probability. Morris (1991, theorem 1a) offered a characterization for the existence of a common prior in no-trade terms, thus providing a variant of the converse statement to the no-trade theorem of Milgrom and Stokey (1982). This result was rediscovered by Feinberg (2000), and a simple geometric proof was given by Samet (1998). There is a fundamental distinction between the common-prior problem and ours. While in the common-prior problem the conditional probability is given, and therefore the full belief hierarchy at every state can be inferred, in our case only the unconditional posterior is considered, and the belief hierarchy is not specified. ${ }^{7}$ Despite this distinction, there is a

${ }^{4}$ Certain compatibility questions for belief hierarchies (without application to the feasibility of joint belief distribution or Bayesian persuasion) were recently addressed by Brooks, Frankel, and Kamenica (2019).

${ }^{5}$ In the example of a polarizing sender that we discuss in sec. IV, receivers have a continuum of actions (the set of actions coincides with the set of possible posterior beliefs), and the above linear-programming approach leads to an infinite-dimensional program.

${ }^{6}$ In sec. IV, we provide several applications of our results and study feasible correlation of posterior beliefs. Related questions arise in numerous papers on information design. See Ely (2017) and Bergemann, Heumann, and Morris (2021).

7 In the notation introduced in our model section below, the common-prior problem can be phrased as follows. Fix a signal space $S_{i}$ for each agent $i$, and denote $\Omega=\prod_{i} S_{i}$. Say that we are given, for each agent $i$ and signal realization $s_{i}$, a conditional distribution $Q_{i}(\cdot)$ supported on the subset of $\Omega$ in which agent $i$ 's signal is $s_{i}$. When does there exist a single probability measure $\mathbb{P}$ on $\Omega$ such that for $\mathbb{P}$-almost every $s_{i}$ it holds that $Q_{s_{i}}(\cdot)=\mathbb{P}\left(\cdot \mid s_{i}\right)$ ? 
connection between the no-trade characterizations of a common prior and of feasible distributions. In a follow-up paper, Morris (2020) demonstrated how to deduce a no-trade characterization of feasibility similar to ours from his earlier characterization of a common prior. This approach leads to a characterization of feasibility for finitely supported distributions and arbitrary finite sets of states. ${ }^{8}$ Morris (2020) also offered a comprehensive discussion of the history of the no-trade approach to the common-prior problem.

Related literature: measures with given marginals.-From a technical perspective, our characterization of feasibility relies on the existence of measures with given marginals. Instead of the classic results of Kellerer (1961) and Strassen (1965) used by Dawid et al. (1995), we apply a more recent result due to Hansel and Troallic (1986). In the economic literature, such tools were applied by Gershkov et al. (2013) and Gershkov, Moldovanu, and Strack (2018). Our feasibility condition for product distributions (proposition 2) shares some similarity with Border's condition of feasibility for reduced-form auctions; see Hart and Reny (2015).

The remainder of the paper is organized as follows. In section II, we introduce the formal problem. In section III, we present our main results. Applications are presented in section IV. In section V, we study first-order Bayesian persuasion and the extreme feasible beliefs. In section VI, we study implementations of a given feasible posterior distribution. Further proofs are provided in the appendix.

\section{Model}

\section{A. Information Structures and Posterior Beliefs}

We consider a binary state space $\Omega=\{\ell, \mathrm{h}\}$ and a set of $n$ agents $N=\{1,2, \ldots, n\}$. An information structure $I=\left(\left(S_{i}\right)_{i \in N}, \mathbb{P}\right)$ consists of signal sets $S_{i}$ (each equipped with a sigma-algebra, which we suppress) for each agent $i$ and a distribution $\mathbb{P} \in \Delta\left(\Omega \times S_{1} \times \cdots \times S_{n}\right)$. Let $\omega, s_{1}, \ldots, s_{n}$ be the random variables corresponding to the $n+1$ coordinates of the underlying space $\Omega \times S_{1} \times \cdots \times S_{n}$. When it is unambiguous, we also use $s_{i}$ to denote a generic element of $S_{i}$.

The prior probability of the high state is denoted by $p=\mathbb{P}(\omega=\mathrm{h})$. Throughout the paper, we assume that $p \in(0,1)$. All $n$ agents initially have prior $p$ regarding the state $\omega$. Then, each agent $i$ observes the signal $s_{i}$. The posterior belief $x_{i}$ attributed to the high state by agent $i$ after receiving the signal $s_{i}$ is

$$
x_{i}=\mathbb{P}\left(\omega=\mathrm{h} \mid s_{i}\right)
$$

\footnotetext{
${ }^{8}$ The restriction to finitely supported distributions can possibly be eliminated by approximation arguments.
} 
We denote by $P_{I}$ the joint distribution of posterior beliefs induced by the information structure $I$. This probability measure on $[0,1]^{N}$ is the joint distribution of $\left(x_{1}, x_{2}, \ldots, x_{n}\right)$. That is, for each measurable $B \subset[0,1]^{N}$,

$$
P_{I}(B)=\mathbb{P}\left(\left(x_{1}, \ldots, x_{n}\right) \in B\right) .
$$

We similarly denote the conditional joint distributions of posterior beliefs by $P_{I}^{\ell}$ and $P_{I}^{\mathrm{h}}$; these are the joint distributions of $\left(x_{1}, \ldots, x_{n}\right)$, conditioned on the state $\omega$.

For a probability measure $P \in \Delta\left([0,1]^{N}\right)$ and for $i \in N$, we denote by $P_{i}$ the marginal distribution, that is, the distribution of the projection on the $i$ th coordinate, or the distribution of the posterior of agent $i$.

\section{B. Feasible Joint Posterior Beliefs}

When is a given distribution on $[0,1]^{N}$ equal to the distribution of posterior beliefs induced by some information structure? This is the main question we study in this paper. The following definition captures this notion formally.

Definition 1. Given $p \in(0,1)$, we say that a distribution $P \in$ $\Delta\left([0,1]^{N}\right)$ is $p$-feasible if there exists some information structure $I$ with prior $p$ such that $P=P_{I}$.

When $p$ is understood from the context, we simply use the term "feasible" rather than " $p$-feasible."

\section{The Single-Agent Case}

Before tackling the question of feasibility for $n$ agents, it is instructive to review the well-understood case of a single agent.

The so-called martingale condition states that the average posterior belief of a feasible posterior distribution is equal to the prior. Formally, given a $p$-feasible distribution $P \in \Delta([0,1])$, it must be the case that

$$
\int_{0}^{1} x \mathrm{~d} P(x)=p .
$$

The necessity of this condition for $p$-feasibility follows from the law of iterated expectation. For the single-agent case, the martingale condition is necessary and sufficient for $P$ to be $p$-feasible. This result is known as the "splitting lemma."

\section{Feasible Joint Beliefs}

\section{A. The Two-Agent Case and the Agreement Theorem}

In the two-agent case, the martingale condition is not sufficient for feasibility. An additional obstruction to feasibility is given by Aumann's 
celebrated agreement theorem (Aumann 1976). We here provide a rephrasing of this theorem in a form that will be useful for us below.

Theorem A (Aumann). Let $\left(\left(S_{i}\right)_{i}, \mathbb{P}\right)$ be an information structure, and let $B_{1} \subseteq S_{1}$ and $B_{2} \subseteq S_{2}$ be subsets of possible signal realizations for agents 1 and 2 , respectively. If

$$
\mathbb{P}\left(s_{1} \in B_{1}, s_{2} \notin B_{2}\right)=\mathbb{P}\left(s_{2} \in B_{2}, s_{1} \notin B_{1}\right)=0,
$$

then

$$
\mathbb{E}\left(x_{1} \cdot \mathbf{1}_{s_{1} \in B_{1}}\right)=\mathbb{E}\left(x_{2} \cdot \mathbf{1}_{s_{2} \in B_{2}}\right) .
$$

To understand why this is a reformulation of the agreement theorem, note that condition (1) implies that

$$
\mathbb{P}\left(s_{1} \in B_{1}, s_{2} \in B_{2}\right)=\mathbb{P}\left(s_{1} \in B_{1}\right)=\mathbb{P}\left(s_{2} \in B_{2}\right),
$$

and thus the event $\left\{s_{1} \in B_{1}, s_{2} \in B_{2}\right\}$ is self-evident, that is, is common knowledge whenever it occurs. ${ }^{9}$ Hence, this form of the agreement theorem states that if agents have common knowledge of the event $\left\{s_{1} \in B_{1}\right.$, $\left.s_{2} \in B_{2}\right\}$, then their average beliefs on this event must coincide. The original theorem follows by choosing $B_{1}$ and $B_{2}$ such that $x_{1}$ is constant on $B_{1}$ and $x_{2}$ is constant on $B_{2}$. The statement of theorem $\mathrm{A}$ is close in form to that of the no-trade theorem of Milgrom and Stokey (1982), which provides the same obstruction to feasibility; we discuss this further below. We do not provide a proof of theorem A, as it is a special case of our quantitative agreement theorem (theorem 1), which we prove below.

Using theorem A, it is easy to construct examples of distributions that are not feasible, even though they satisfy the martingale condition. For example, consider the prior $p=1 / 2$ and the distribution $P=(1 / 2) \delta_{0,1}+$ $(1 / 2) \delta_{1,0}$, where $\delta_{x 1, x 2}$ denotes the point mass at $\left(x_{1}, x_{2}\right)$. Clearly, $P$ satisfies the martingale condition. Nevertheless, it is not feasible, since under this distribution agents "agree to disagree," when agent 1 attributes probability 1 to the high state and agent 2 considers the probability of this state to be 0 . Indeed, assume toward a contradiction that $P$ is equal to $P_{I}$, for some information structure $I=\left(\left(S_{i}\right)_{i}, \mathbb{P}\right)$. Then, the events $B_{1}=\left\{s \in S_{1}\right.$ : $\left.x_{1}(s)=1\right\}$ and $B_{2}=\left\{s \in S_{2}: x_{2}(s)=0\right\}$ satisfy the common-knowledge condition (1). But

$$
\mathbb{P}\left(\omega=\mathrm{h} \mid s_{1} \in B_{1}\right)=1 \neq 0=\mathbb{P}\left(\omega=\mathrm{h} \mid s_{2} \in B_{2}\right),
$$

in contradiction to theorem A.

This example can be extended to a more general necessary condition for feasibility of a joint posterior distribution.

\footnotetext{
${ }^{9}$ An event is self-evident if, whenever it occurs, all agents almost surely know that it has occurred. An event $A$ is common knowledge at an outcome $a \in A$ if $A$ contains a selfevident event that contains $a$.
} 
Corollary A. Let $P \in \Delta\left([0,1]^{2}\right)$ be feasible for some $p$, and let $A_{1}$ and $A_{2}$ be measurable subsets of $[0,1]$. Denote complements by $\overline{A_{i}}=$ $[0,1] \backslash A_{i}$. If

$$
P\left(A_{1} \times \overline{A_{2}}\right)=P\left(\overline{A_{1}} \times A_{2}\right)=0,
$$

then

$$
\int_{A_{1}} x \mathrm{~d} P_{1}(x)=\int_{A_{2}} x \mathrm{~d} P_{2}(x)
$$

Corollary $\mathrm{A}$ is a recasting of theorem A into a direct condition for feasibility: condition (2) states that the event $A_{1} \times A_{2}$ is self-evident: it has the same probability as $A_{1}$ and the same probability as $A_{2}$. And each of the two integrals in equation (3) is equal to the average belief of agent $i$ conditioned on $A_{i}$, times the probability of $A_{i}$. Hence, corollary A follows immediately from theorem A, by setting $B_{i}=\left\{s_{i}: x_{i}\left(s_{i}\right) \in A_{i}\right\}$ for $i=1,2$. The advantage of this formulation is that it takes the form of a direct condition on $P .^{10}$

\section{B. A Quantitative Agreement Theorem}

While Aumann's agreement theorem provides an obstruction to feasibility, a joint posterior distribution can be infeasible even when it does not imply that agents agree to disagree. A larger set of necessary conditions follows from our first result, which is a quantitative version of the agreement theorem.

Theorem 1. Let $\left(\left(S_{i}\right)_{i}, \mathbb{P}\right)$ be an information structure, and let $B_{1} \subseteq S_{1}$ and $B_{2} \subseteq S_{2}$ be sets of possible signal realizations for agents 1 and 2, respectively. Then,

$$
\mathbb{P}\left(s_{1} \in B_{1}, s_{2} \notin B_{2}\right) \geq \mathbb{E}\left(x_{1} \cdot \mathbf{1}_{s_{1} \in B_{1}}\right)-\mathbb{E}\left(x_{2} \cdot \mathbf{1}_{s_{2} \in B_{2}}\right) \geq-\mathbb{P}\left(s_{2} \in B_{2}, s_{1} \notin B_{1}\right) .
$$

Proof. By the law of total expectations, we have that

$$
\mathbb{E}\left(x_{i} \cdot \mathbf{1}_{s_{i} \in B_{i}}\right)=\mathbb{E}\left(\mathbb{P}\left(\omega=\mathrm{h} \mid s_{i}\right) \cdot \mathbf{1}_{s_{i} \in B_{i}}\right)=\mathbb{P}\left(\omega=\mathrm{h}, s_{i} \in B_{i}\right) .
$$

We thus need to show that

$$
\begin{aligned}
\mathbb{P}\left(s_{1} \in B_{1}, s_{2} \notin B_{2}\right) & \geq \mathbb{P}\left(\omega=\mathrm{h}, s_{1} \in B_{1}\right)-\mathbb{P}\left(\omega=\mathrm{h}, s_{2} \in B_{2}\right) \\
& \geq-\mathbb{P}\left(s_{2} \in B_{2}, s_{1} \notin B_{1}\right) .
\end{aligned}
$$

\footnotetext{
${ }^{10}$ A particular case of corollary A appears in Dawid et al. (1995, theorem 5.2), and the inaccuracy in that statement was later corrected by Burdzy and Pitman (2020, proposition 2.1). Levy, Moreno de Barreda, and Razin (2020) found a necessary condition for feasibility equivalent to corollary A for distributions with finite support. None of these papers mentions the connection to the agreement theorem.
} 
We show the first inequality; the second follows by an identical argument. We in fact demonstrate a stronger inequality:

$\mathbb{P}\left(\omega=\mathrm{h}, s_{1} \in B_{1}, s_{2} \notin B_{2}\right) \geq \mathbb{P}\left(\omega=\mathrm{h}, s_{1} \in B_{1}\right)-\mathbb{P}\left(\omega=\mathrm{h}, s_{2} \in B_{2}\right)$.

Denote the conditional probability $\mathbb{P}(C \mid \omega=\mathrm{h})$ by $\mathbb{P}^{\mathrm{h}}(C)$ for any event $C$. Then, inequality (4) is equivalent to the elementary inequality $\mathbb{P}^{\mathrm{h}}(A \cap$ $\bar{B}) \geq \mathbb{P}^{\mathrm{h}}(A)-\mathbb{P}^{\mathrm{h}}(B)$, which holds for any pair of events $A$ and $B$ and any probability measure $\mathbb{P}^{\mathrm{h}}$. QED

A comparison to the agreement theorem (theorem A) is illustrative. In theorem A, the common-knowledge assumption (1) implies equality of average posteriors. Here, condition (1) has been removed, and we instead bound the difference in the average posteriors by the extent to which (1) is violated. Thus, one can think of theorem 1 as quantifying the extent to which approximate common knowledge implies approximate agreement. The agreement theorem becomes the special case in which condition (1) holds. ${ }^{11}$

In analogy to corollary A, we use theorem 1 to derive further necessary conditions for feasibility.

Corollary 1 . Let $P \in \Delta\left([0,1]^{2}\right)$ be $p$-feasible for some $p$, and let $A_{1}$ and $A_{2}$ be measurable subsets of $[0,1]$. Then,

$$
P\left(A_{1} \times \overline{A_{2}}\right) \geq \int_{A_{1}} x \mathrm{~d} P_{1}(x)-\int_{A_{2}} x \mathrm{~d} P_{2}(x) \geq-P\left(\overline{A_{1}} \times A_{2}\right) .
$$

Corollary 1 admits a simple interpretation in terms of the no-trade theorem (Milgrom and Stokey 1982). Consider three risk-neutral agents: two traders and a mediator. Trader 2 owns a good with an unknown quality $\omega \in\{0,1\}$. The mediator also owns a copy of the same good. The two traders receive private information regarding the quality of the good, with a joint belief distribution $P \in \Delta\left([0,1]^{2}\right)$. The mediator knows $P$ and the realized pair $\left(x_{1}, x_{2}\right)$.

Let $A_{1}, A_{2} \subseteq[0,1]$ be any measurable sets and consider the following trading scheme. The mediator buys the good from trader 2 whenever $x_{2} \in A_{2}$ at a price of $x_{2}$. The mediator sells one copy of the good to trader 1 whenever $x_{1} \in A_{1}$ at a price of $x_{1}$. Thus, the mediator may need to use her copy of the good, in case she sells but does not buy.

We argue that the mediator's expected profit is at least

$$
\int_{A_{1}} x \mathrm{~d} P_{1}(x)-\int_{A_{2}} x \mathrm{~d} P_{2}(x)-P\left(A_{1} \times \overline{A_{2}}\right) .
$$

\footnotetext{
${ }^{11}$ An alternative approach to quantitative extensions of the agreement theorem is by the concept of common p-beliefs (Monderer and Samet 1989). Neeman (1996) showed that when posteriors are common $p$-beliefs, they cannot differ by more than $1-p$. However, we are not aware of any formal connection between this extension and our theorem 2.
} 
The first two addends correspond to the expected transfer between each trader and the mediator. The last addend corresponds to the event that the mediator has to sell his own good to trader 1, since trader 2's belief $x_{2}$ is not in $A_{2}$ and trader 1's belief is in $A_{1}$. In this case, the mediator loses at most 1 .

Clearly, the mediator does not provide any additional information to the two players, and so their expected profit is zero. Thus, the mediator's expected profit is also zero, and so we have arrived at the left-hand inequality of (5). The right-hand inequality follows by symmetry.

\section{A Characterization for Two Agents}

Dawid et al. (1995) characterized the feasible distributions for the case of two agents, by applying a result of Kellerer (1961) and Strassen (1965). Although they do not relate their result to the agreement theorem or phrase it in these terms, what they show is that the condition of feasibility from corollary 1 is both necessary and sufficient.

Theorem 2 (Dawid et al.). A probability measure $P \in \Delta\left([0,1]^{2}\right)$ is $p$-feasible for some $p$ if and only if

$$
P\left(A_{1} \times \overline{A_{2}}\right) \geq \int_{A_{1}} x \mathrm{~d} P_{1}(x)-\int_{A_{2}} x \mathrm{~d} P_{2}(x) \geq-P\left(\overline{A_{1}} \times A_{2}\right)
$$

for all measurable $A_{1}, A_{2} \subseteq[0,1]$.

The necessity of condition (6) is corollary 1 . The sufficiency requires another argument, which uses a theorem of Kellerer (1961). For the reader's convenience, we present the complete proof of theorem 2 in appendix A.

It follows from theorem 2 and the single-agent martingale condition that when condition (6) holds, $P$ is $p$-feasible for

$$
p=\int_{0}^{1} x \mathrm{~d} P_{1}(x)=\int_{0}^{1} x \mathrm{~d} P_{2}(x) .
$$

We note that in Dawid et al. (1995), condition (6) was written in the equivalent form

$$
P\left(A_{1} \times B_{2}\right)+p \geq \int_{A_{1}} x \mathrm{~d} P_{1}(x)+\int_{B_{2}} x \mathrm{~d} P_{2}(x),
$$

from which the relation to the agreement theorem is harder to see.

It is natural to wonder whether condition (6) can be relaxed to a simpler sufficient condition and in particular whether it suffices to check it on $A_{1}, A_{2}$ that are intervals. As we show in appendix $\mathrm{D}$, restricting condition (6) to intervals results in a condition that is not sufficient: we construct a measure that is not feasible but satisfies condition (5) for all intervals $A_{1}, A_{2}$. The constructed measure demonstrates that the condition derived by Ziegler (2020) for feasibility is necessary but insufficient. 
D. A Characterization for Any Number of Agents

For three or more agents, theorem 2 provides a necessary condition for feasibility, as the joint belief distribution of each pair of agents must clearly satisfy condition (6). However, this condition is not sufficient: we construct below an example of three agents whose belief distribution satisfies condition (6) for each pair of agents and yet is not feasible. The violation of feasibility stems from a violation of the no-trade theorem, in a manner similar to the one illustrated above for two agents. We use this approach to provide a necessary and sufficient condition for feasibility for an arbitrary number of agents.

A trading scheme consists of $n$ measurable functions $a_{i}:[0,1] \rightarrow[-1,1]$, $i=1, \ldots, n$. Given agent $i$ s posterior $x_{i}$, a mediator sells $a_{i}\left(x_{i}\right)$ units of the good to agent $i$ for the price of $x_{i}$ per unit, so that the total transfer is $a_{i}\left(x_{i}\right) x_{i}$.

Clearly, each agent's expected profit is zero, since she is buying or selling the good at her expected value. Hence, the mediator's expected profit is also zero. We argue that

$$
\int_{[0,1]^{n}}\left(\sum_{i=1}^{n} a_{i}\left(x_{i}\right) x_{i}-\max \left\{0, \sum_{i=1}^{n} a_{i}\left(x_{i}\right)\right\}\right) \mathrm{d} P\left(x_{1}, \ldots, x_{n}\right)
$$

is a lower bound on the mediator's profit. Indeed, the first addend in the integral is the total transfer to the mediator. The second is equal to the total number of units of the good that the mediator needs to contribute to the transaction, in case the total amount that she sells exceeds the total amount that she buys. Since each unit is worth at most 1 , she loses at most $\sum a_{i}\left(x_{i}\right)$, whenever this sum is positive. Thus, since the mediator's profit is zero, it follows that ( 7$)$ cannot be positive if $P$ is feasible.

Our characterization shows that this condition is also sufficient for feasibility. The proof is given in appendix B.

Theorem 3. A probability measure $P \in \Delta\left([0,1]^{n}\right)$ is $p$-feasible for some $p$ if and only if, for every trading scheme $\left(a_{1}, \ldots, a_{n}\right)$,

$$
\int_{[0,1]^{n}}\left(\sum_{i=1}^{n} a_{i}\left(x_{i}\right) x_{i}-\max \left\{0, \sum_{i=1}^{n} a_{i}\left(x_{i}\right)\right\}\right) \mathrm{d} P\left(x_{1}, \ldots, x_{n}\right) \leq 0 .
$$

In the case of two agents, by taking $a_{i}= \pm \mathbf{1}_{A_{i}}$ in condition (8), we recover condition (6) from theorem 2 . However, we are not aware of a simple argument for deducing condition (8) from condition (6) in the two-agent case. In particular, theorem 2 is not a simple corollary of theorem 3 , since the latter requires a broader set of trading schemes, while indicators are enough for the former. Relatedly, Kellerer's theorem that underlies theorem 2 holds only for $n=2$ and cannot be extended to the multidimensional case without expanding the set of test functions; see the discussion in Strassen (1965, 436-37). 
A natural question is whether theorem 3 can be strengthened, along the lines of theorem 2, to consider only indicator trading schemes: Is it sufficient to consider trading schemes of the form $a_{i}= \pm \mathbf{1}_{A_{i}}$, so that each agent is either a buyer or a seller and has a set of beliefs in which she trades one unit? By computerized verification, it is possible to show that the answer is no. A counterexample is the distribution $\nu \times \nu \times$ $\nu \in \Delta\left([0,1]^{3}\right)$, where $\nu=(1 / 3)\left(\delta_{3 / 14}+\delta_{1 / 2}+\delta_{11 / 14}\right)$. This distribution is not feasible, and yet each of the small number of possible indicator trading schemes is not profitable.

Since $\nu \times \nu$ is feasible, this example also shows that theorem 3 provides additional obstructions for feasibility when $n \geq 3$, beyond the pairwise condition implied by theorem 2 . We end this section with another such example. Consider three agents whose beliefs $\left(x_{1}, x_{2}, x_{3}\right)$ are distributed uniformly and independently on $[0,1]$. That is, let their joint belief distribution $P \in \Delta\left([0,1]^{3}\right)$ be the Lebesgue measure. By proposition 2 below, the agents pairwise satisfy the condition of theorem 2 . We argue that this is nevertheless not a feasible distribution.

To see this, consider the trading scheme given by

$$
a_{1}(x)=a_{2}(x)=a_{3}(x)=\mathbf{1}_{x \geq 2 / 3}-\mathbf{1}_{x \leq 1 / 3} .
$$

In this scheme, each agent buys a unit whenever her belief is above $2 / 3$ and sells when it is below $1 / 3$. A simple calculation shows that condition (8) of theorem 3 is violated. ${ }^{12}$ These examples illustrate the general phenomenon that is captured by proposition 3 below: for any distribution $\nu$ on $[0,1]$ not concentrated at one point, $\nu^{n}$ becomes infeasible for large enough $n$. In these two examples $n=3$ suffices.

\section{Applications}

\section{A. Identically Distributed Binary Signals}

As an illustration of the restrictions imposed by the requirement of feasibility, consider a setting with prior $p=1 / 2$ and two agents who each receive a binary signal that equals the state with some probability $r>1 / 2$. What joint distributions are feasible?

The canonical setting is the one in which signals are independent, conditioned on the state. Note that they are not unconditionally independent: while each agent has each posterior with probability $1 / 2$, conditioned on

${ }^{12}$ We get $\int_{[0,1]^{3}} a_{i}\left(x_{i}\right) x_{i} \mathrm{~d} P=-\int_{0}^{1 / 3} x \mathrm{~d} x+\int_{2 / 3}^{1} x \mathrm{~d} x=2 / 9$. Hence, $\int_{[0,1]^{3}} \sum_{i=1}^{3} a_{i}\left(x_{i}\right) x_{i} \mathrm{~d} P=6 / 9$. The hyperplanes $x_{i} \in\{1 / 3,2 / 3\}$ partition $[0,1]^{3}$ into 27 small cubes. There is one small cube where the sum $\sum_{i=1}^{3} a_{i}\left(x_{i}\right)$ is equal to 3 , three cubes where the sum equals 2 , and six cubes where it has the value of 1 ; on the other cubes it is nonpositive. Hence, $\int_{[0,1]^{3}} \max \left\{0, \sum_{i=1}^{3} a_{i}\left(x_{i}\right)\right\} \mathrm{d} P=15 / 27=5 / 9$. We see that condition (8) is violated and conclude that the uniform distribution on $[0,1]^{3}$ is infeasible. We thank Eric Neyman for alerting us to an error in a previous version of this example. 
the first agent acquiring a high posterior, the second agent is more likely to also have a high posterior than a low one. In this case, the induced belief distribution is

$$
P=\frac{r^{2}+(1-r)^{2}}{2}\left[\delta_{r, r}+\delta_{1-r, 1-r}\right]+r(1-r)\left[\delta_{1-r, r}+\delta_{r, 1-r}\right] .
$$

Another simple case is the one in which both agents observe the same signal, in which case the posteriors are of course perfectly correlated, and the distribution is

$$
P=\frac{1}{2} \delta_{r, r}+\frac{1}{2} \delta_{1-r, 1-r},
$$

as in the single-agent case. In both of these cases, $P$ is feasible, since we derive it from a joint signal distribution.

The case in which agents' posteriors are perfectly anticorrelated, that is,

$$
P=\frac{1}{2} \delta_{r, 1-r}+\frac{1}{2} \delta_{1-r, r}
$$

is precluded by the agreement theorem and its corollary A, as agents here agree to disagree on their posteriors.

More generally, we can consider the case in which, conditioned on an agent's posterior, the other agent has the same posterior with probability $c$. That is,

$$
P=\frac{c}{2}\left[\delta_{r, r}+\delta_{1-r, 1-r}\right]+\frac{1-c}{2}\left[\delta_{1-r, r}+\delta_{r, 1-r}\right] .
$$

In this case, there is never common knowledge of beliefs, as long as $c<1$. The natural question is, To what extent can the signals be anticorrelated? Can they, for example, be (unconditionally) independent, so that the probability that an agent, after observing a signal, assigns to the other agent's posterior is still uniform over $\{r, 1-r\}$ ? A common intuition suggests that this is impossible, since even if signals are independent, given the state, the induced unconditional distribution of posteriors inherits the dependence on the state, and thus the posteriors must be dependent; on the other hand, if the conditional distribution of signals is the same in both states, they convey no information, and thus the posterior just equals the prior. Perhaps surprisingly, this intuition is wrong, and posteriors can be independent and even anticorrelated; see, for example, Burdzy and Pitman (2020).

Proposition 1. A joint belief distribution $P$, as given in equation (9), is $1 / 2$-feasible if and only if $c \geq 2 r-1$.

This proposition follows directly from theorem 2: one needs only to check the sets $A_{i}=\{r\}$ and $A_{i}=\{1-r\}$. More generally, for finitely supported $P$ only finitely many conditions need be checked. 
Proposition 1 implies that indeed too much anticorrelation is infeasible, especially as the signals become more informative. Nevertheless, it is possible that the agents' posteriors are independent of each other (i.e., $c=1 / 2$ ) as long as $r \leq 3 / 4$. Moreover, for $r<3 / 4$, the posteriors can be negatively correlated; for example, $P$ is feasible for $c=1 / 3$ and $r=2 / 3$. In this case, posteriors are either $1 / 3$ or $2 / 3$, each obtained with probability $1 / 2$. When an agent has the high posterior, she assigns probability $2 / 3$ to the event that the other agent has the low posterior.

\section{B. Unconditionally Independent Signals}

As another application of theorem 2, we further explore independent joint posterior belief distributions. To motivate this application, consider a sender (e.g., a consulting agency) who wants to reveal some information to receivers (its clients). However, there is an additional concern: all of the receivers must be unable to form any nontrivial guess about the information received by their counterpart. This can be motivated either by privacy concerns or by the desire to avoid complicated strategic reasoning on receivers' side. For example, consider the case that the receivers are two firms competing on the same market and plan to use the received information to adjust their strategies. If their posteriors are not independent, they might engage in a complicated reasoning involving higher-order beliefs, as in Weinstein and Yildiz (2007). Another motivation for studying independent joint beliefs comes from mechanism design, where these distributions arise endogenously (see, e.g., Bergemann, Brooks, and Morris 2017; Brooks and Du 2019).

We saw above that identically distributed binary signals can be independent for prior $p=1 / 2$ as long as $r \leq 3 / 4$. As a second example, let $P$ be the uniform distribution on the unit square. Following Gutmann et al. (1991), we verify that it is $1 / 2$-feasible and find the information structure inducing it. This distribution clearly satisfies the martingale condition, so it remains to check that

$$
P\left(A_{1} \times \overline{A_{2}}\right) \geq \int_{A_{1}} x \mathrm{~d} P_{1}(x)-\int_{A_{2}} x \mathrm{~d} P_{2}(x) .
$$

The other inequality of condition (6) will follow by symmetry.

Let $1-a$ be the Lebesgue measure of $A_{1}$ and $b$ that of $A_{2}$. Then, the left-hand side equals $(1-a)(1-b)$, and the right-hand side is maximized when $A_{1}$ is pushed as much as possible toward high values of the integrand (i.e., $\left.A_{1}=[a, 1]\right)$ and $A_{2}$ is pushed toward low values $\left(A_{2}=\right.$ $[0, b])$. We get the following inequality:

$$
(1-a)(1-b) \geq \int_{a}^{1} x \mathrm{~d} x-\int_{0}^{b} x \mathrm{~d} x=\frac{1}{2}\left(1-a^{2}-b^{2}\right) .
$$


Simplifying the expression, we get $(a+b-1)^{2} \geq 0$, which holds for any $a$ and $b$. Thus, the uniform distribution is $1 / 2$-feasible.

The equality attained at $a+b-1=0$ helps us guess the information structure that induces this distribution of beliefs. Comparing the inequality in the statement of theorem 1 to inequality (4), we see that for any such information structure, $\mathbb{P}\left(\omega=\mathrm{h}, x_{1} \in[a, 1], x_{2} \in[b, 1]\right)=\mathbb{P}\left(x_{1} \in[a, 1]\right.$, $\left.x_{2} \in[b, 1]\right)$ for $a+b-1=0$, and hence $\mathbb{P}\left(\omega=\mathrm{h} \mid x_{1} \in[a, 1], x_{2} \in\right.$ $[b, 1])=1$. In other words, whenever the pair of posteriors is in the triangle $T=\left\{\left(x_{1}, x_{2}\right) \in[0,1]^{2}: x_{1}+x_{2}>1\right\}$, the state is $\omega=\mathrm{h}$; by the symmetric argument, $\omega=\ell$ whenever posteriors are in $[0,1]^{2} \backslash T$. Hence, one can use the following information structure: sample a pair of signals $\left(s_{1}, s_{2}\right)$ uniformly from $T$ if $\omega=\mathrm{h}$ and from $[0,1]^{2} \backslash T$ if $\omega=\ell$. This information structure leads to $x_{i}\left(s_{i}\right)=s_{i}$ and thus induces the uniform distribution. In section VI, we describe a family of information structures inducing the uniform distribution on $[c, 1-c]^{2}$.

We generalize this example to study the conditions for $1 / 2$-feasibility of more general product distributions. The next proposition provides a necessary and sufficient condition for feasibility of a large class of such distributions and shows that the uniform distribution is, in fact, an important edge case. We say that a distribution $\nu \in \Delta([0,1])$ is symmetric around $1 / 2$ if its cumulative distribution function $F(a)=\nu([0, a])$ satisfies $F(a)=1-F(1-a)$ for all $a \in[0,1]$. Recall that $\mu \in \Delta([0,1])$ is a mean-preserving spread of $\mu^{\prime} \in \Delta([0,1])$ if there exist random variables $x$, $x^{\prime}$ such that $x \sim \mu, x^{\prime} \sim \mu^{\prime}$, and $\mathbb{E}\left(x \mid x^{\prime}\right)=x^{\prime} .{ }^{13}$

Proposition 2. Let $P=\nu \times \nu$, where $\nu \in \Delta([0,1])$ is symmetric around $1 / 2$. Then, $P$ is $1 / 2$-feasible if and only if the uniform distribution on $[0,1]$ is a mean-preserving spread of $\nu$.

In particular, among symmetric, 1/2-feasible product distributions, the uniform is maximal in the convex order. ${ }^{14}$

The proof of proposition 2 is relegated to appendix C. The "if" direction is a consequence of the following, more general lemma.

Lemma 1. Let $P=\mu_{1} \times \cdots \times \mu_{n} \in \Delta\left([0,1]^{n}\right)$ be $p$-feasible, and let $P^{\prime}=$ $\mu_{1}^{\prime} \times \cdots \times \mu_{n}^{\prime}$, where each $\mu_{i}$ is a mean-preserving spread of $\mu_{i}^{\prime}$. Then, $P^{\prime}$ is also $p$-feasible.

${ }^{13}$ Equivalently, $\int f(x) \mathrm{d} \mu(x) \geq \int f(x) \mathrm{d} \mu^{\prime}(x)$ for every bounded convex $f$. Another equivalent condition is that both have the same expectation and that $\int_{0}^{y} F(x) \mathrm{d} x \leq \int_{0}^{y} F^{\prime}(x) \mathrm{d} x$ for all $y \in[0,1]$, where $F$ and $F^{\prime}$ are the cumulative distribution functions of $\mu$ and $\mu^{\prime}$, respectively. See Blackwell (1953).

${ }^{14}$ We note that the symmetry assumption cannot be dropped. Indeed, the uniform distribution is not a mean-preserving spread of the nonsymmetric distribution $\nu=a$. $\delta_{1-a}+(1-a) \cdot \delta_{1}$ with $a=1 / \sqrt{2}$, but nevertheless $\nu \times \nu$ is feasible. Feasibility can checked via theorem 2 or directly by constructing the information structure: both agents have two signals $S_{1}=S_{2}=\{\underline{s}, \bar{s}\}$; if the state is $\omega=\mathrm{h}$, then the pair of signals is $(\bar{s}, \bar{s})$ with probability $2(1-a)^{2}$ and $(\underline{s}, \bar{s})$ or $(\bar{s}, \underline{s})$ with probabilities $(1 / 2)-(1-a)^{2}$; if the state is $\omega=\ell$, the signals are always $(\underline{s}, \underline{s})$. The uniform distribution is not a mean-preserving spread of $\nu$ since $\int_{1 / 2}^{1}(x-(1 / 2)) \mathrm{d} v(x)=(1 / 2)-[1 /(2 \sqrt{2})]>\int_{1 / 2}^{1}(x-(1 / 2)) \mathrm{d} x=1 / 8$. 
This lemma can be seen as a corollary of Blackwell's theorem (Blackwell 1951, theorem 12): given an information structure that induces $P$, one can apply an independent garbling to each coordinate to arrive at a structure that induces $P^{\prime}$. Our proof illustrates how the result can be obtained via the no-trade arguments of our theorem 3, without invoking Blackwell's theorem.

Using proposition 2, we show in appendix $\mathrm{F}$ that Gaussian signals may induce independent beliefs, provided that they are not too informative. Let $\nu$ be the belief distribution induced by a signal that, conditioned on the state, is a unit variance Gaussian with mean $\pm d$. Then, $\nu \times \nu$ is $1 / 2$ feasible if and only if $d$ lies between the $1 / 4$ quantile and the $3 / 4$ quantile of the standard normal distribution.

Interestingly, product distributions with a given marginal cease being feasible once the number of agents becomes large enough, as demonstrated in the following proposition.

Proposition 3. For every probability measure $\nu \in \Delta([0,1])$ that differs from a Dirac measure, for sufficiently large $n$ the product distribution $\nu^{n} \in \Delta\left([0,1]^{n}\right)$ is not feasible.

The proof, which is relegated to appendix $\mathrm{C}$, uses our theorem 3 . We show that with a sufficiently large number of agents, a mediator can implement a strictly beneficial trading scheme, that is, one that violates theorem 3. In fact, we show that the product distribution $\nu^{n}$ is infeasible whenever $\lfloor n / 2\rfloor>(1 / 8)\left(\int_{m}^{1} x \mathrm{~d} v(x)-\int_{0}^{m} x \mathrm{~d} v(x)\right)^{-2}$, where $m$ is the median of the marginal distribution $\nu$.

\section{First-Order Bayesian Persuasion and Extreme Feasible Joint Posterior Belief Distributions}

\section{A. First-Order Bayesian Persuasion}

In this section, we consider a sender who sends information regarding an underlying state to a group of $n$ receivers. The sender's utility depends on the actions of the receivers. We assume that each receiver's utility depends only on the state and her own action, as, for example, is common in the social learning literature. Since the equilibrium action of a receiver is dictated solely by her first-order beliefs, we call this setting "first-order Bayesian persuasion." We note that the results of this section do not rely on the characterizations of feasibility given in theorems 2 and 3 , but rather offer an additional set of tools to study the set of feasible distributions of posteriors.

Formally, a first-order Bayesian persuasion problem is given by $B=$ $\left(N, p,\left(A_{i}\right)_{i \in N},\left(u_{i}\right)_{i \in N}, u_{\mathrm{s}}\right)$. As above, $\omega \in\{\ell, \mathrm{h}\}$ is a binary state for which $n=|N|$ receivers have a common prior $p \in(0,1)$. Each receiver $i \in N$ has to choose an action $a_{i}$ from a compact metric set of actions $A_{i}$. Her 
utility $u_{i}\left(\omega, a_{i}\right)$ depends only on the state and her action. A single sender has utility $u_{\mathrm{s}}\left(a_{1}, \ldots, a_{n}\right)$, which depends on the receivers' actions.

The sender chooses an information structure $I=\left(\left(S_{i}\right)_{i \in N}, \mathbb{P}\right)$ with prior $p$, and each receiver $i$ observes a private signal $s_{i} \in S_{i}$ and then chooses an action $a_{i}$

In equilibrium, the action

$$
\tilde{a}_{i} \in \underset{a_{i} \in A_{i}}{\operatorname{argmax}} \mathbb{E}\left(u_{i}\left(\omega, a_{i}\right) \mid s_{i}\right)
$$

is chosen by $i$ to maximize her expected utility conditioned on her information $s_{i}$. Note that since $u_{i}$ depends only on $\omega$ and $a_{i}$, it follows that, given the information structure $I$, receiver $i$ s posterior $x_{i}=\mathbb{P}\left(\omega=\mathrm{h} \mid s_{i}\right)$ is a sufficient statistic for her utility, that is,

$$
\max _{a_{i} \in A_{i}} \mathbb{E}\left(u_{i}\left(\omega, a_{i}\right) \mid s_{i}\right)=\max _{a_{i} \in A_{i}} \mathbb{E}\left(u_{i}\left(\omega, a_{i}\right) \mid x_{i}\right),
$$

and so a receiver does not decrease her expected utility by discarding her private signal, retaining only the first-order posterior belief $x_{i}$. We accordingly consider only equilibria in which the agents - even when they are indifferent - use only $x_{i}$ to choose their actions, so that $\tilde{a}_{i}$ is a function of $x_{i}{ }^{15}$

The information structure $I$ is chosen to maximize the expectation of $u_{\mathrm{s}}$. We assume that $u_{i}$ and $u_{\mathrm{s}}$ are upper-semicontinuous, to ensure the existence of equilibria. The value $V(B)$ is the sender's expected equilibrium utility in the first-order Bayesian persuasion problem $B$.

A crucial feature is the assumption that $u_{i}$ does not depend on the other agents' actions. The case in which externalities are allowed is the general problem of Bayesian mechanism design, which is beyond the scope of this paper. ${ }^{16}$ In contrast, in first-order Bayesian persuasion the receivers have no strategic incentives. This implies that their higher-order beliefs are irrelevant to the sender, who in turn is solely interested in their first-order posterior beliefs. This is captured by the following proposition, which states that for every first-order Bayesian persuasion problem there is an indirect utility function that the sender maximizes by choosing the receivers' posteriors. Of course, the posterior distribution must be feasible.

Denote by $\mathcal{P}_{p}^{N}$ the set of $p$-feasible $P \in \Delta\left([0,1]^{N}\right)$.

Proposition 4. For every first-order Bayesian persuasion problem $B=$ $\left(N, p,\left(A_{i}\right)_{i},\left(u_{i}\right)_{i}, u_{\mathrm{s}}\right)$, there is an indirect utility function $v:[0,1]^{N} \rightarrow \mathbb{R}$, such that the value $V(B)$ is given by

${ }_{15}$ This refinement rules out equilibria in which, e.g., an agent uses higher-order beliefs to break ties when indifferent between two actions.

${ }^{16}$ Nevertheless, our approach remains applicable even in the presence of externalities if the game played by receivers is "simple" in the sense of Börgers and Li (2019), i.e., the equilibrium behavior of a receiver does not require her to form higher-order beliefs. 


$$
V(B)=\max _{P \in \mathcal{P}_{p}^{n}} \int v\left(x_{1}, \ldots, x_{n}\right) \mathrm{d} P\left(x_{1}, \ldots, x_{n}\right) .
$$

\section{B. Example: Polarizing Receivers}

In section IV, we showed that joint belief distributions can be anticorrelated and explored the extent to which identically distributed binary signals can be anticorrelated. For general signal distributions, this question can be formalized using a first-order Bayesian persuasion approach.

Consider a sender whose indirect utility for the posteriors of two receivers is

$$
v\left(x_{1}, x_{2}\right)=\left|x_{1}-x_{2}\right|^{a},
$$

for some parameter $a>0$. Informally, the sender wishes to maximize the polarization between receivers or the discrepancy between their posteriors.

For the case $a=2$, we solve the sender's problem completely.

Proposition 5. Let $V$ be the value of the two receiver first-order Bayesian persuasion problem where the sender's indirect utility is

$$
v\left(x_{1}, x_{2}\right)=\left(x_{1}-x_{2}\right)^{2}
$$

and the prior is $p \in(0,1)$. Then, $V(B)=(1-p) p$.

In this case of $a=2$, the optimum can be achieved by completely informing one agent and leaving the other completely uninformed, for example, by letting $s_{1}=\omega$ and $s_{2}=0$. We show in appendix $\mathrm{C}$ that when $p=1 / 2$, the same policy is also optimal for all $a<2$. Dubins and Pitman (1980) show the same result for $a=1$ and any $p$.

For $a \geq 3$, it is no longer true that it is optimal to reveal the state to one receiver and leave the other uninformed, which yields utility $2^{-a}$ to the sender. For example, the posterior distribution

$$
P=\frac{1}{4} \delta_{0,2 / 3}+\frac{1}{4} \delta_{2 / 3,0}+\frac{1}{2} \delta_{2 / 3,2 / 3}
$$

can be easily verified to be $1 / 2$-feasible, using condition (6), and yields utility $(1 / 2)(2 / 3)^{a}$, which for $a=3$ (for example) is larger than $2^{-a}$.

Another approach to quantifying the extent to which beliefs can be anticorrelated is by directly minimizing their covariance. This question admits a simple, nontrivial solution, as the next proposition shows: for the prior $p=1 / 2$, the smallest possible covariance between posterior beliefs is $-1 / 32$, and it is achieved on a distribution supported on four points.

Proposition 6. Let $V$ be the value of the two-receiver first-order Bayesian persuasion problem where the prior is $p=1 / 2$ and the sender's indirect utility is

$$
v\left(x_{1}, x_{2}\right)=-\left(x_{1}-p\right) \cdot\left(x_{2}-p\right) .
$$


Then, $V(B)=1 / 32$ and is achieved by

$$
P=\frac{1}{8} \delta_{3 / 4,0}+\frac{3}{8} \delta_{3 / 4,1 / 2}+\frac{1}{8} \delta_{1 / 4,1}+\frac{3}{8} \delta_{1 / 4,1 / 2} .
$$

The distribution $P$ is depicted in figure 1 .

The proofs of propositions 5 and 6 are presented in appendix C. They do not rely on our characterization of feasible beliefs and instead use the fact that conditional expectations are orthogonal projections in the Hilbert space of square-integrable random variables. This technique exploits the quadratic form of these persuasion problems. A natural avenue for future research is the extension of these techniques - or the development of new techniques - to tackle nonquadratic problems.

\section{Extreme Feasible Joint Posterior Belief Distributions}

The set $\mathcal{P}_{p}^{N}$ of $p$-feasible joint posterior belief distributions is a convex, compact subset of $\Delta\left([0,1]^{N}\right)$, when the latter is naturally equipped with the weak* topology; see Dubins and Pitman (1980) or proposition 8 in the appendix. Together with proposition 4, this fact implies that $V(B)$ is always achieved at an extreme point of the set of $p$-feasible distributions $\mathcal{P}_{p}^{N}$. It is thus natural to study the set of extreme points.

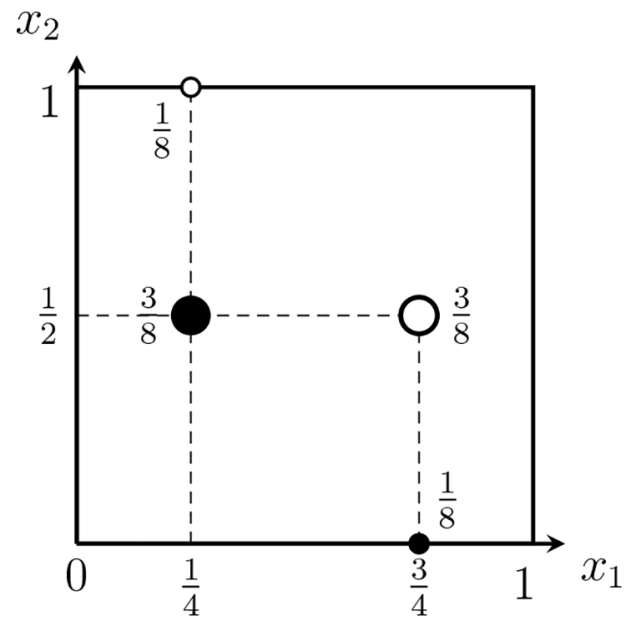

FIG. 1.-Optimal distribution of the first-order Bayesian persuasion problem given by the indirect utility $v\left(x_{1}, x_{2}\right)=-\left(x_{1}-p\right) \cdot\left(x_{2}-p\right)$ and prior $p=1 / 2$; see proposition 6 . For $p=1 / 2$, this distribution achieves the lowest possible covariance between posterior beliefs: $-1 / 32$. Filled circles describe the distribution of posteriors conditional on $\omega=\ell$. Open circles describe the distribution of posteriors conditional on $\omega=\mathrm{h}$. 
In the single-agent case, the concavification argument of Aumann and Maschler (1995) and Kamenica and Gentzkow (2011) implies that every extreme point is a distribution with support of size at most 2. This is not true for two or more agents. For example, the posterior distribution with support of size 3 defined in equation (10) is extreme in $\mathcal{P}_{1 / 2}^{2}$, since its restriction to any support of smaller cardinality is not feasible, as it cannot satisfy the martingale condition. The next theorem shows that there in fact exist extreme points with countably infinite support. It also states that the support cannot be too large, in the sense that every extreme point is supported on a set with a Lebesgue measure of zero.

Theorem 4. Let $|N| \geq 2$. Then, (1) for every $p \in(0,1)$ there exists an extreme point in $\mathcal{P}_{p}^{N}$ whose support has an infinite countable number of points; and (2) for every extreme $P \in \mathcal{P}_{p}^{N}$ there exists a measurable $A \subseteq[0,1]^{N}$ such that $P(A)=1$, and the Lebesgue measure of $A$ is zero.

To prove the first part of theorem 4, we explicitly construct an extreme feasible belief distribution with countably infinite support; see appendix C. The construction is a variant of Rubinstein's email game (Rubinstein 1989). Unlike Rubinstein's email game, no agent in our construction is fully informed about the state. The resulting belief distribution for two agents is depicted in figure 2. Interestingly, it is possible to modify our construction in a way that places the beliefs closer and closer to the diagonal. This results in a sequence of extreme points that converge to a distribution that is supported on the diagonal and has support size larger than 2. Every extreme point that is supported on the diagonal is supported on at most

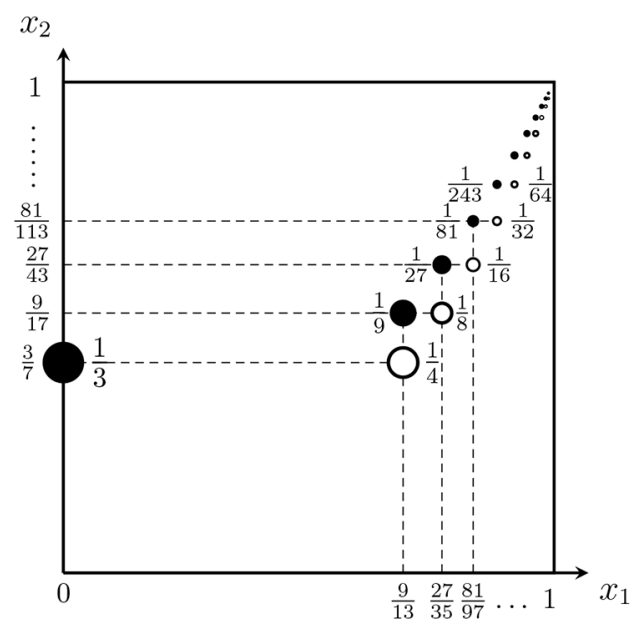

FIG. 2.-An extreme point of $\mathcal{P}_{1 / 2}^{2}$ with infinite countable support. The numbers near the points indicate their probabilities. Conditional on $\omega=\ell$, the pair of posteriors belongs to the set of filled circles. Conditional on $\omega=\mathrm{h}$, the pair of posteriors belongs to the set of open circles. 
two points, since this reduces to the single-agent case. Therefore, for two agents or more, the set of extreme points is not closed within the set of feasible distributions. This demonstrates another distinction from the singleagent case where the set of extreme points is closed.

The proof of the second part of theorem 4 relies on the classic result of Lindenstrauss (1965) regarding extreme points of the set of joint distributions with given marginals.

Theorem 4 leaves a natural question open: Are there any nonatomic extreme points? Or, conversely, does every extreme point have countable support?

\section{Implementing Feasible Distributions}

Assume that we are given a feasible distribution $P \in \Delta\left([0,1]^{n}\right)$. A natural question is, Which information structures induce $P$ ? And, relatedly, which conditional posterior belief distributions $\left(P^{\ell}, P^{\mathrm{h}}\right)$ are compatible with $P$ ?

By lemma 3 (stated in the appendix), $P$ is feasible if and only if there exists a distribution $Q \in \Delta\left([0,1]^{n}\right)$ such that $Q \leq(1 / p) P$ and $\mathrm{d} Q_{i}(x)=$ $(x / p) \mathrm{d} P_{i}(x)$ for all agents $i$. Note that this is a linear program, which in general is infinite dimensional.

Given a solution $Q$ define $P^{\mathrm{h}}=Q$ and $P^{\ell}=(P-p \cdot Q) /(1-p)$. Then, the distribution of posteriors $P$ is induced by the information structure in which the signals have joint distribution $P^{\omega}$ conditional on $\omega$ (see lemma 2 and its proof); in this case, we say that $P$ is implemented by the pair $\left(P^{\ell}, P^{\mathrm{h}}\right)$, which are also the conditional distributions of the beliefs. Thus, to find an information structure that induces $P$, it suffices to solve the above-mentioned linear program.

When $P$ has finite support, this linear program has finite dimension, and thus a solution can be numerically calculated by simply applying a linear program solver. In the general, infinite-dimensional case, we do not expect that simple, closed-form solutions always exist. An exception is the single-agent case, in which an implementing pair is given by (see lemma 2)

$$
\begin{aligned}
\mathrm{d} P^{\ell}(x) & =\frac{1-x}{1-p} \mathrm{~d} P(x) \text { and } \\
\mathrm{d} P^{\mathrm{h}}(x) & =\frac{x}{p} \mathrm{~d} P(x) .
\end{aligned}
$$

We make two observations about the single-agent case. First, there is a unique pair of conditional belief distributions $\left(P^{\ell}, P^{\mathrm{h}}\right)$ that implements $P$ : every information structure that induces $P$ will have the same conditional distributions of beliefs. Second, the two distributions $P^{\ell}$ and $P^{\mathrm{h}}$ have the Radon-Nikodym derivative $\left(\mathrm{d} P^{\mathrm{h}} / \mathrm{d} P^{\ell}\right)(x)=[(1-p) / p][x /(1-x)]$ and so are mutually absolutely continuous, unless there is an atom on 0 
or 1 . As we now discuss, neither of these two properties holds in general beyond the single-agent case.

We consider the case that the number of agents is $n \geq 2$ and that $P \in \Delta\left([0,1]^{n}\right)$ is a feasible distribution that admits a density. In this case, the next proposition shows that $P$ can be implemented by $\left(P^{\ell}, P^{\mathrm{h}}\right)$ that are very far from being mutually absolutely continuous: they are supported on disjoint sets. ${ }^{17}$

Proposition 7. Let $n \geq 2$, and let $P \in \Delta\left([0,1]^{n}\right)$ be $p$-feasible for some $p$. Assume that $P$ admits a density. Then, there exists a subset $D \subset[0,1]^{n}$, such that $P$ can implemented by $\left(P^{\ell}, P^{\mathrm{h}}\right)$ with the property that $P^{\mathrm{h}}$ is supported on $D$ and $P^{\ell}$ is supported on the complement $\bar{D}$. Furthermore, restricted to $D$ and $\bar{D}$ respectively, $P^{\ell}=[1 /(1-p)] P$, and $P^{\mathrm{h}}=(1 / p) P$.

Proof. Let $R$ be a measure on $[0,1]^{n}$. For a subset $D \subseteq[0,1]^{n}$, we denote by $\left.R\right|_{D}$ the restriction of $R$ to this subset, that is, $\left.R\right|_{D}(A)=$ $R(D \cap A)$ for any measurable set $A$.

In theorem 3 in Gutmann et al. (1991), it was shown that for any absolutely continuous, finite measure $R$ on $[0,1]^{n}$ and every measure $Q \leq R$, there exists a measurable set $D$ such that $\left.R\right|_{D}$ and $Q$ have identical marginals.

Apply this result to $R=(1 / p) P$ and $Q \leq(1 / p) P$, which solves the linear program of lemma 3. Then, there is a $D \subset[0,1]^{n}$ such that $Q$ and $\left.(1 / p) P\right|_{D}$ have the same marginals. Let $Q^{\prime}=\left.(1 / p) P\right|_{D}$. Then, $Q^{\prime}$ is also a solution to the linear program of lemma 3: $Q^{\prime} \leq(1 / p) P$ and $\mathrm{d} Q_{i}(x)=(x / p) \mathrm{d} P_{i}(x)$. Hence, $P$ is implemented by $P^{\mathrm{h}}=Q^{\prime}=\left.(1 / p) P\right|_{D}$ and $P^{\ell}=\left(P-p \cdot Q^{\prime}\right) /$ $(1-p)=\left.[1 /(1-p)] P\right|_{\bar{D}}$, which proves the claim. QED

When $n \geq 2$, an implementation is not always unique, in contrast to the single-agent case. To see this, consider the case of $n=2$ and $P$ equal to the uniform distribution $U_{S}$ on the square $S=[c, 1-c]^{2}$ with $c \in[0,1 / 2)$. It is $1 / 2$-feasible, since the marginals are second-order dominated by the uniform distribution on $[0,1]$ (see proposition 2 ). In section II, we saw the implementation of the uniform distribution on $[0,1]^{2}$, which corresponds to $c=0$. For $c>0$, that implementation suggests that solutions of the linear program of lemma 3 might be found in the form of a convex combination of $U_{S}$ and the uniform distribution $U_{T}$ on the upper triangle $T=S \cap\left\{x_{1}+x_{2} \geq 1\right\}$. Indeed, it is easy to check that

$$
Q=\frac{1-c}{1+c} \cdot U_{T}+\frac{2 c}{1+c} \cdot U_{S}
$$

\footnotetext{
17 These pairs are also easily seen to be extreme points among the convex set of pairs that implement $P$.
} 
2570

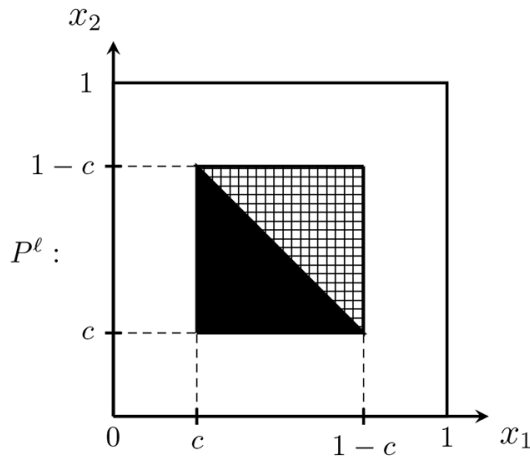

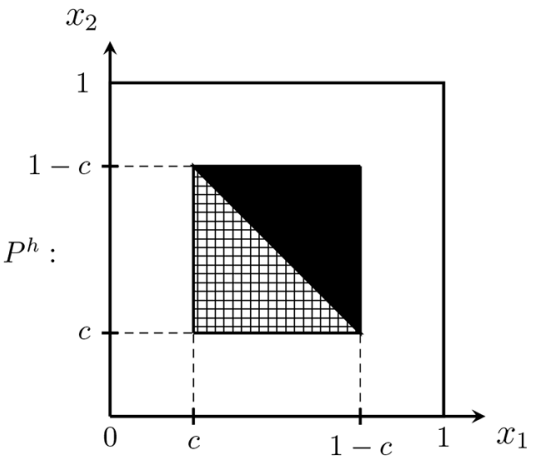

Fig. 3. - The implementation $\left(P^{\ell}, P^{\mathrm{h}}\right)$ of the uniform distribution on $[c, 1-c]^{2}$. Black and cross-hatched areas correspond to higher and lower densities, respectively.

has the correct marginals and satisfies $Q \leq 2 \cdot U_{S}$. The corresponding pair of distributions $\left(P^{\ell}, P^{\mathrm{h}}\right)$ is illustrated in figure 3 .

Note that in this implementation the two distributions $\left(P^{\ell}, P^{\mathrm{h}}\right)$ do not have disjoint supports. Hence, proposition 7 implies that another implementation exists. This is illustrated in figure 4 .

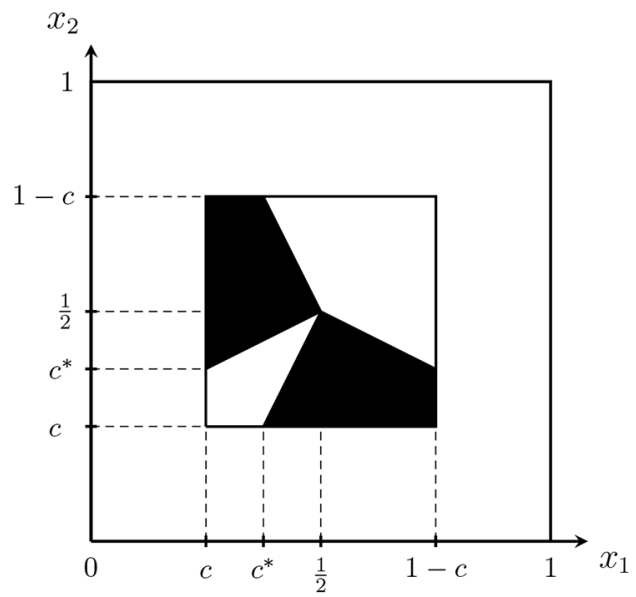

FIG. 4.-An implementation of the uniform distribution on $[c, 1-c]^{2}$ in which $P^{\ell}$ (uniform over the black area) and $P^{\mathrm{h}}$ (uniform over the white subset of $[c, 1-c]^{2}$ ) have disjoint supports. We denote $c^{*}=2 c(1-c)$. 


\section{Appendix A}

\section{Proof of Theorem 2}

In this appendix, we present a proof of theorem 2, a result due to Dawid et al. (1995). We begin with the following simple lemma, which gives a direct revelation principle for joint belief distributions. Similar statements have appeared in the literature (e.g., Gutmann et al. 1991; Dawid et al. 1995; Arieli, Babichenko, and Smorodinsky 2020; Levy, Moreno de Barreda, and Razin 2020; Ziegler 2020). We include a proof for the convenience of the reader.

Lemma 2. Let $P \in \Delta\left([0,1]^{n}\right)$ be a $p$-feasible belief distribution. Then, there exist $P^{\ell}, P^{\mathrm{h}} \in \Delta\left([0,1]^{n}\right)$ such that $P=(1-p) \cdot P^{\ell}+p \cdot P^{\mathrm{h}}$ and for every $i \in$ $\{1, \ldots, n\}$ the marginal distributions $P_{i}^{\mathrm{h}}$ and $P_{i}$ satisfy

$$
\mathrm{d} P_{i}^{\mathrm{h}}(x)=\frac{x}{p} \mathrm{~d} P_{i}(x)
$$

In particular, every feasible belief distribution can be induced by an information structure in which, for each $i$, the belief $x_{i}$ is equal to the signal $s_{i}$.

The next lemma gives a necessary and sufficient condition for feasibility in terms of the existence of a measure with given marginals and a given upper bound.

Lemma 3. For $n \geq 2$ agents, a distribution $P \in \Delta\left([0,1]^{n}\right)$ is $p$-feasible if and only if there exists a probability measure $Q \in \Delta\left([0,1]^{n}\right)$ such that

1. $Q$ is upper-bounded by $(1 / p) P$, that is, $Q(A) \leq(1 / p) P(A)$ for any measurable $A \subseteq[0,1]^{n}$, and

2. for every $i \in\{1, \ldots, n\}$, the marginal distribution $Q_{i}$ is given by

$$
\mathrm{d} Q_{i}(x)=\frac{x}{p} \mathrm{~d} P_{i}(x) .
$$

Proof. If $P$ is feasible, then by lemma 2 there is a pair $P^{\ell}, P^{\mathrm{h}} \in \Delta\left([0,1]^{n}\right)$ such that $P=(1-p) P^{\ell}+p \cdot P^{\mathrm{h}}$ and such that equation (A1) holds. Picking $Q=P^{\mathrm{h}}$, it follows from equation (A1) that $Q \leq(1 / p) P$ and that $Q$ has the desired marginals. In the opposite direction, if there is $Q$ satisfying the theorem hypothesis, then let $P^{\mathrm{h}}=Q$ and $P^{\ell}=(P-p \cdot Q) /(1-p)$. Consider the information structure $I$ in which $\omega=\mathrm{h}$ with probability $p$, and $\left(s_{1}, \ldots, s_{n}\right)$ is chosen from $P^{\mathrm{h}}$ conditioned on $\omega=\mathrm{h}$, and from $P^{\ell}$ conditioned on $\omega=\ell$. It follows from equation (A2) that $P$ is the joint posterior distribution induced by $I$ and is hence $p$-feasible. QED

Lemma 3 reduces the question of feasibility to the question of the existence of a bounded measure with given marginals. This is a well-studied question in the case $n=2$. When the upper bound is proportional to the Lebesgue measure, Lorentz (1949) provided the answer in terms of first-order stochastic dominance of marginals. The discrete analog of this result for matrices is known as the GaleRyser theorem (Gale 1957; Ryser 1957). The condition for general upper-bound measures was derived by Kellerer (1961, Satz 4.2); the formulation below is due to Strassen (1965, theorem 6).

Theorem B (Kellerer, Strassen). Fix $P \in \Delta\left([0,1]^{2}\right), 0<p \leq 1$, and $M_{1}$, $M_{2} \in \Delta([0,1])$. Then, the following are equivalent. 
1. There exists a probability measure $Q \in \Delta\left([0,1]^{2}\right)$ that is bounded from above by $(1 / p) P$ and has marginals $Q_{i}=M_{i}$.

2. For every measurable $B_{1}, B_{2} \subset[0,1]$, it holds that

$$
\frac{1}{p} P\left(B_{1} \times B_{2}\right) \geq M_{1}\left(B_{1}\right)+M_{2}\left(B_{2}\right)-1 .
$$

As we now show, theorem 2 is a consequence of Kellerer's theorem and lemma $3 .^{18}$

Proof of theorem 2.-When $P$ is $p$-feasible, condition (6) follows immediately from corollary 1 . To prove the other direction, we assume that $P$ satisfies condition (6) and prove that $P$ is $p$-feasible for some $p$. To this end, we use Kellerer's theorem to show that there exists a $Q \in \Delta\left([0,1]^{2}\right)$ satisfying the conditions of lemma 3 .

Assume, then, that $P$ satisfies condition (6); that is, for every measurable $A_{1}, A_{2} \subseteq[0,1]$, it holds that

$$
P\left(A_{1} \times \overline{A_{2}}\right) \geq \int_{A_{1}} x \mathrm{~d} P_{1}(x)-\int_{A_{2}} x \mathrm{~d} P_{2}(x) \geq P\left(\overline{A_{1}} \times A_{2}\right) .
$$

Applying this to $A_{1}=A_{2}=[0,1]$ yields

$$
\int_{0}^{1} x \mathrm{~d} P_{1}(x)=\int_{0}^{1} x \mathrm{~d} P_{2}(x) .
$$

We accordingly set $p=\int_{0}^{1} x \mathrm{~d} P_{1}(x)$ and define the measures $M_{1}, M_{2} \in \Delta([0,1])$ by $\mathrm{d} M_{i}(x)=(x / p) \mathrm{d} P_{i}(x)$. Given any two measurable subsets $B_{1}, B_{2} \subseteq[0,1]$, let $A_{1}=$ $B_{1}$ and $A_{2}=\overline{B_{2}}$. Applying the left-hand inequality of condition (A4) to $A_{1}=B_{1}$ and $A_{2}=\overline{B_{2}}$ yields

$$
P\left(B_{1} \times B_{2}\right) \geq \int_{B_{1}} x \mathrm{~d} P_{1}(x)-\int_{\overline{B_{2}}} x \mathrm{~d} P_{2}(x) .
$$

Since $\mathrm{d} M_{i}(x)=(x / p) \mathrm{d} P_{i}(x)$, we can write this as

$$
P\left(B_{1} \times B_{2}\right) \geq p \cdot M_{1}\left(B_{1}\right)-p \cdot M_{2}\left(\overline{B_{2}}\right) ;
$$

dividing by $p$ and substituting $M_{2}\left(\overline{B_{2}}\right)=1-M_{2}\left(B_{2}\right)$ yields

$$
\frac{1}{p} P\left(B_{1} \times B_{2}\right) \geq M_{1}\left(B_{1}\right)+M_{2}\left(B_{2}\right)-1 .
$$

Thus condition (A3) holds, and we can directly apply Kellerer's theorem to conclude that there exists a measure $Q$ that satisfies the conditions of lemma 3 , as condition (12) is simply $Q_{i}=M_{i}$. Hence, $P$ is $p$-feasible. QED

\section{Appendix B}

\section{Proof of Theorem 3}

In this appendix, we prove theorem 3 . The proof of necessity is straightforward and explained before the theorem statement. The proof of sufficiency uses théorème 2.6 of Hansel and Troallic (1986), which is a generalization of Kellerer's theorem.

\footnotetext{
${ }_{18}$ We note that this result can also be proved by interpreting the infinite-dimensional linear program for $Q$ from lemma 3 as a condition that there is a feasible flow of magnitude 1 in an auxiliary network (as in Gale 1957, but with a continuum of edges) and then using max-flow/min-cut duality for infinite networks (Neumann 1985).
} 
Recall that a paving of a set $X$ is a set of subsets of $X$ that includes the empty set and that an algebra is a paving that is closed under unions and complements. By a collection we mean a multiset; that is, the same element can enter the collection several times. Denote $\overline{\mathbb{R}}_{+}=[0, \infty]$. A finitely additive measure is a map from an algebra to $\overline{\mathbb{R}}_{+}$that is additive for disjoint sets.

Theorem C. (Hansel and Troallic 1986). Let $X$ be a set and $\mathcal{F}$ an algebra of subsets of $X$. Let $\mathcal{F}_{1}, \ldots, \mathcal{F}_{n}$ and $\mathcal{G}_{1}, \ldots, \mathcal{G}_{m}$ be subpavings of $\mathcal{F}$. For $i \in\{1, \ldots, n\}$ and $j \in\{1, \ldots, m\}$, let $\alpha_{i}: F_{i} \rightarrow \overline{\mathbb{R}}_{+}$and $\beta_{j}: \mathcal{G}_{j} \rightarrow \overline{\mathbb{R}}_{+}$, respectively, be maps that vanish on the empty set. Then, the following are equivalent.

1. There is a finitely additive measure $Q: \mathcal{F} \rightarrow \mathbb{R}_{+}$such that, for every $i \in\{1, \ldots, n\}$ and every $A \in \mathcal{F}_{i}$, it holds that $\alpha_{i}(A) \leq Q(A)$ and, for every $j \in\{1, \ldots, m\}$ and every $B \in \mathcal{G}_{j}$, it holds that $Q(B) \leq \beta_{j}(B)$.

2. For every finite collection of sets $\mathcal{A}_{1} \subseteq \mathcal{F}_{1}, \ldots, \mathcal{A}_{n} \subseteq \mathcal{F}_{n}, \mathcal{B}_{1} \subseteq \mathcal{G}_{1}, \ldots$, $\mathcal{B}_{m} \subseteq \mathcal{G}_{m}$, if

$$
\sum_{i=1}^{n} \sum_{A \in \mathcal{A}_{i}} \mathbf{1}_{A} \leq \sum_{j=1}^{m} \sum_{B \in \mathcal{B}_{j}} \mathbf{1}_{B}
$$

then

$$
\sum_{i=1}^{n} \sum_{A \in \mathcal{A}_{i}} \alpha_{i}(A) \leq \sum_{j=1}^{m} \sum_{B \in \mathcal{B}_{j}} \beta_{j}(B) .
$$

We will need the following corollary of this result.

Corollary C. Fix $P \in \Delta\left([0,1]^{n}\right), 0<p \leq 1$, and $M_{1}, \ldots, M_{n} \in \Delta([0,1])$. Then, the following are equivalent.

1. There exists a probability measure $Q \in \Delta\left([0,1]^{n}\right)$ that is upper-bounded by $(1 / p) P$ and has marginals $Q_{i}=M_{i}$.

2. For every $\mathcal{A}_{1}, \ldots, \mathcal{A}_{n}$ - finite collections of Borel subsets of $[0,1]$ - every $\mathcal{C}$-a finite collection of Borel subsets of $[0,1]^{n}$ - and every nonnegative integer $K$, if

$$
\sum_{i=1}^{n} \sum_{A \in \mathcal{A}_{i}} \mathbf{1}_{A}\left(x_{i}\right) \leq K+\sum_{C \in \mathcal{C}} \mathbf{1}_{C}\left(x_{1}, \ldots, x_{n}\right)
$$

for all $\left(x_{1}, \ldots, x_{n}\right)$, then

$$
\sum_{i=1}^{n} \sum_{A \in \mathcal{A}_{i}} M_{i}(A) \leq K+\sum_{C \in \mathcal{C}} \frac{1}{p} P(C) .
$$

Proof. The proof that part 1 implies part 2 is simple and omitted. ${ }^{19}$

Let $\mathcal{F}$ be the Borel sigma-algebra of $[0,1]^{n}$. For each $i \in\{1, \ldots, n\}$, let $\mathcal{F}_{i}$ be the sub-sigma-algebra of sets that are measurable in the $i$ th coordinate. That is, $\mathcal{F}_{i}$ consists of sets of the form $[0,1]^{i-1} \times A \times[0,1]^{n-i}$, where $A$ is any Borel subset of $[0,1]$. Denote by $\pi_{i}:[0,1]^{n} \rightarrow[0,1]$ the projection on the $i$ th coordinate.

Let $m=2$. Define $\mathcal{G}_{i}$ as the trivial algebra $\left\{\varnothing,[0,1]^{n}\right\}$ and $\mathcal{G}_{2}=\mathcal{F}$. Let $\alpha_{i}=M_{i}$, $\beta_{1}\left([0,1]^{n}\right)=1, \beta_{1}(\varnothing)=0$, and $\beta_{2}=(1 / p) P$. Let $\mathcal{A}_{1}, \ldots, \mathcal{A}_{n}$ and $\mathcal{C}$ satisfy condition (B1). By abuse of notation we identify each $A \in \mathcal{A}_{i}$ with its preimage

${ }^{19}$ We make use of only the other, nontrivial direction. 
$\pi_{i}^{-1}(A)=[0,1]^{i-1} \times A \times[0,1]^{n-i}$. Thus, $\mathcal{A}_{i} \subseteq \mathcal{F}_{i}$. We define $\mathcal{B}_{1}$ and $\mathcal{B}_{2}$ as follows. The collection $\mathcal{B}_{1} \subseteq \mathcal{G}_{1}$ contains $K$ copies of $[0,1]^{n}$, and $\mathcal{B}_{m}=\mathcal{C} \subseteq \mathcal{G}_{2}$.

We can apply theorem $\mathrm{C}$ directly to conclude that there is a finitely additive measure $Q$ that is upper-bounded by $(1 / p) P$ and has $Q\left([0,1]^{n}\right) \leq 1$ and whose marginals $Q_{i}$ bound $M_{i}$ from above for $i \in N$. Each $M_{i}$ is a probability measure, and the total mass of $Q$ is at most 1 ; hence, $Q_{i}$ can upper-bound $M_{i}$ only if $Q_{i}=M_{i}$. We conclude that $Q$ is a probability measure with marginals $M_{i}$ for each $i$. Since $Q$ is upper-bounded by a sigma-additive measure, it is also itself sigmaadditive (see lemma 4). QED

Lemma 4. Let $\mathcal{F}$ be a sigma-algebra of subsets of $X$. Let $\mu: \mathcal{F} \rightarrow \overline{\mathbb{R}}_{+}$be a finitely additive measure, and let $\nu: \mathcal{F} \rightarrow \overline{\mathbb{R}}_{+}$be a sigma-additive measure. If $\mu \leq \nu$ and $\nu(X)<\infty$, then $\mu$ is sigma-additive.

Proof. Let $A_{1}, A_{2}, \ldots \in \mathcal{F}$ be pairwise disjoint. Denote $A=\cup_{i} A_{i}$. Then, by additivity, we have that

$$
\mu(A)=\mu\left(\cup_{i=1}^{n} A_{i}\right)+\mu\left(\cup_{i=n+1}^{\infty} A_{i}\right) \geq \mu\left(\cup_{i=1}^{n} A_{i}\right)=\sum_{i=1}^{n} \mu\left(A_{i}\right) .
$$

Hence, $\mu(A) \geq \sum_{i=1}^{\infty} \mu\left(A_{i}\right)$. For the other direction,

$$
\mu(A)=\mu\left(\cup_{i=1}^{n} A_{i}\right)+\mu\left(\cup_{i=n+1}^{\infty} A_{i}\right) \leq \sum_{i=1}^{n} \mu\left(A_{i}\right)+\nu\left(\cup_{i=n+1}^{\infty} A_{i}\right),
$$

since $\mu \leq \nu$. By the sigma-additivity and finiteness of $\nu$, the last addend vanishes as $n$ tends to infinity. Hence, $\mu(A) \leq \sum_{i=1}^{\infty} \mu\left(A_{i}\right)$. QED

We are now ready to finish the proof of theorem 3. We show that condition (8) suffices for feasibility by checking that it implies the conditions of lemma 3 . Assume, then, that $P$ satisfies condition (8); that is, for every trading scheme $\left(a_{1}, \ldots, a_{i}\right)$ it holds that

$$
\int_{[0,1]^{n}}\left(\sum_{i=1}^{n} a_{i}\left(x_{i}\right) x_{i}-\max \left\{0, \sum_{i=1}^{n} a_{i}\left(x_{i}\right)\right\}\right) \mathrm{d} P\left(x_{1}, \ldots, x_{n}\right) \leq 0 .
$$

Choose $i, j \in\{1, \ldots, n\}$, and consider the trading scheme in which $a_{i}=1, a_{j}=$ -1 , and $a_{k}=0$ for all $k \notin\{i, j\}$. Then, condition (B3) implies that $\int_{0}^{1} x \mathrm{~d} P_{i}(x)=$ $\int_{0}^{1} x \mathrm{~d} P_{j}(x)$. We accordingly set $p=\int_{0}^{1} x \mathrm{~d} P_{i}(x)$ and note that this definition is independent of the choice of $i$. Define the measures $M_{i} \in \Delta\left([0,1]^{n}\right)$ by $\mathrm{d}_{i}(x)=$ $(x / p) \mathrm{d} P_{i}(x)$

Let $\mathcal{A}_{1}, \ldots, \mathcal{A}_{n}, K$, and $\mathcal{C}$ satisfy condition (B1). We will show that condition (B2) must hold. This will conclude the proof, since then it follows from corollary $\mathrm{C}$ that there exists a measure $Q$ that satisfies the conditions of lemma 3 , as condition (A2) is simply $Q_{i}=M_{i}$.

To show that condition (B2) holds, define the trading scheme $\left(a_{1}, \ldots, a_{n}\right)$ by

$$
a_{i}(x)=c \cdot\left(\sum_{A \in \mathcal{A}_{i}} \mathbf{1}_{A}(x)-\frac{K}{n}\right),
$$

where $c$ is a normalization constant chosen small enough so that the image of all the $a_{\mathrm{i}}$ is in $[-1,1]$. Then, by condition (B3),

$$
\int_{[0,1]^{n}} \sum_{i=1}^{n} a_{i}\left(x_{i}\right) x_{i} \mathrm{~d} P\left(x_{1}, \ldots, x_{n}\right) \leq \int_{[0,1]^{n}} \max \left\{0, \sum_{i=1}^{n} a_{i}\left(x_{i}\right)\right\} \mathrm{d} P\left(x_{1}, \ldots, x_{n}\right) .
$$


We substitute the definition of the trading scheme and the measures $M_{i}$. This yields

$$
p \cdot \sum_{i=1}^{n} \sum_{A \in \mathcal{A}_{i}} M_{i}(A)-p \cdot K \leq \int_{[0,1]^{n}} \max \left\{0, \sum_{i=1}^{n} \sum_{A \in \mathcal{A}_{i}} \mathbf{1}_{A}\left(x_{i}\right)-K\right\} \mathrm{d} P\left(x_{1}, \ldots, x_{n}\right) .
$$

By condition (B1), the integrand on the right-hand side is at most $\Sigma_{C \in C} \mathbf{1}_{C}\left(x_{1}, \ldots, x_{n}\right)$. Hence,

$$
p \cdot \sum_{i=1}^{n} \sum_{A \in \mathcal{A}_{i}} M_{i}(A)-p \cdot K \leq \sum_{C \in \mathcal{C}} P(C) .
$$

Dividing by $p$ and rearranging yields

$$
\sum_{i=1}^{n} \sum_{A \in \mathcal{A}_{i}} M_{i}(A) \leq K+\sum_{C \in \mathcal{C}} \frac{1}{p} P(C) .
$$

Thus, condition (B2) holds, and by lemma 3 the distribution $P$ is $p$-feasible. This completes the proof of theorem 3 .

We end this appendix by noting a different avenue for proving this theorem. Lemma 3 gives a necessary and sufficient condition for a distribution $P$ to be feasible, in terms of the existence of a distribution $Q$ satisfying two properties. Since the support of $Q$ is always a subset of the support of $P$, for finitely supported $P$ the conditions of the lemma reduce to a finite number of equalities and inequalities on a finite number of variables. Theorem 3 then becomes an corollary of the Farkas lemma. ${ }^{20}$ Theorem 3 for general distributions can then be deduced by approximation arguments.

\section{Appendix C}

\section{Additional Missing Claims and Proofs}

\section{C1. Proof of Lemma 1}

To prove the lemma, we assume that $P^{\prime}$ is not $p$-feasible and show that $P$ is not $p$-feasible.

Let $\left(x_{1}, x_{1}^{\prime}, \ldots, x_{n}, x_{n}^{\prime}\right)$ be random variables such that (1) each pair $\left(x_{i}, x_{i}^{\prime}\right)$ is independent of the rest, (2) $x_{i}$ has distribution $\mu_{i}$ and $x_{i}^{\prime}$ has distribution $\mu_{i}^{\prime}$, and (3) $\mathbb{E}\left(x_{i} \mid x_{i}^{\prime}\right)=x_{i}^{\prime}$, which is possible because $\mu_{i}$ is a mean-preserving spread of $\mu_{i}^{\prime}$.

By this definition, $P$ is the joint distribution of $\left(x_{1}, \ldots, x_{n}\right)$ and $P^{\prime}$ is the joint distribution of $\left(x_{1}^{\prime}, \ldots, x_{n}^{\prime}\right)$. Hence, by theorem 3 , in order to prove our claim it suffices to find a trading scheme $\left(a_{1}, \ldots, a_{n}\right)$ such that

$$
\mathbb{E}\left(\sum_{i=1}^{n} a_{i}\left(x_{i}\right) x_{i}-\max \left\{0, \sum_{i=1}^{n} a_{i}\left(x_{i}\right)\right\}\right)>0,
$$

which would violate condition (8).

${ }^{20}$ A similar approach to the characterization of finitely supported feasible distributions was taken by Morris (2020). 
Since $P^{\prime}$ is not $p$-feasible, it follows from theorem 3 that there exists a trading scheme $\left(a_{1}^{\prime}, \ldots, a_{n}^{\prime}\right)$ such that

$$
\mathbb{E}\left(\sum_{i=1}^{n} a_{i}^{\prime}\left(x_{i}^{\prime}\right) x_{i}^{\prime}-\max \left\{0, \sum_{i=1}^{n} a_{i}^{\prime}\left(x_{i}^{\prime}\right)\right\}\right)>0 .
$$

Define $a_{i}$ by $^{21}$

$$
a_{i}\left(x_{i}\right)=\mathbb{E}\left(a_{i}^{\prime}\left(x_{i}^{\prime}\right) \mid x_{i}\right) .
$$

It follows from the definition of $a_{i}$, from the law of iterated expectation, and from $\mathbb{E}\left(x_{i} \mid x_{i}^{\prime}\right)=x_{i}^{\prime}$ that

$$
\begin{aligned}
\mathbb{E}\left(a_{i}\left(x_{i}\right) \cdot x_{i}\right) & =\mathbb{E}\left(\mathbb{E}\left(a_{i}^{\prime}\left(x_{i}^{\prime}\right) \mid x_{i}\right) \cdot x_{i}\right) \\
& =\mathbb{E}\left(\mathbb{E}\left(a_{i}^{\prime}\left(x_{i}^{\prime}\right) \cdot x_{i} \mid x_{i}\right)\right) \\
& =\mathbb{E}\left(a_{i}^{\prime}\left(x_{i}^{\prime}\right) \cdot x_{i}\right) \\
& =\mathbb{E}\left(\mathbb{E}\left(a_{i}^{\prime}\left(x_{i}^{\prime}\right) \cdot x_{i} \mid x_{i}^{\prime}\right)\right) \\
& =\mathbb{E}\left(a_{i}^{\prime}\left(x_{i}^{\prime}\right) \cdot \mathbb{E}\left(x_{i} \mid x_{i}^{\prime}\right)\right) \\
& =\mathbb{E}\left(a_{i}^{\prime}\left(x_{i}^{\prime}\right) \cdot x_{i}^{\prime}\right) .
\end{aligned}
$$

In addition, we have

$$
\begin{aligned}
\mathbb{E}\left(-\max \left\{0, \sum_{i=1}^{n} a_{i}\left(x_{i}\right)\right\}\right) & =\mathbb{E}\left(-\max \left\{0, \sum_{i=1}^{n} \mathbb{E}\left(a_{i}^{\prime}\left(x_{i}^{\prime}\right) \mid x_{i}\right)\right\}\right) \\
& =\mathbb{E}\left(-\max \left\{0, \mathbb{E}\left(\sum_{i=1}^{n} a_{i}^{\prime}\left(x_{i}^{\prime}\right) \mid x_{1}, \ldots, x_{n}\right)\right\}\right) \\
& \geq \mathbb{E}\left(\mathbb{E}\left(-\max \left\{0, \sum_{i=1}^{n} a_{i}^{\prime}\left(x_{i}^{\prime}\right)\right\} \mid x_{1}, \ldots, x_{n}\right)\right) \\
& =\mathbb{E}\left(-\max \left\{0, \sum_{i=1}^{n} a_{i}^{\prime}\left(x_{i}^{\prime}\right)\right\}\right),
\end{aligned}
$$

where the first equality holds by the definition of $a_{i}$, the second equality holds because $\left(x_{1}, \ldots, x_{n}\right)$ are independent, the third inequality follows from Jensen's inequality because the function $x \mapsto-\max \{0, x\}$ is concave, and the last equality is again the law of iterated expectation.

Together, equations (C3) and (C4) imply that the left-hand side of (C1) is at least as large as that of (C2). Since the latter is positive, we conclude that

$$
\mathbb{E}\left(\sum_{i=1}^{n} a_{i}\left(x_{i}\right) x_{i}-\max \left\{0, \sum_{i=1}^{n} a_{i}\left(x_{i}\right)\right\}\right) \geq \mathbb{E}\left(\sum_{i=1}^{n} a_{i}^{\prime}\left(x_{i}^{\prime}\right) x_{i}^{\prime}-\max \left\{0, \sum_{i=1}^{n} a_{i}^{\prime}\left(x_{i}^{\prime}\right)\right\}\right)>0,
$$

as desired. QED

${ }^{21}$ This defines only $a_{i} \mu_{i}$-almost everywhere, which is sufficient for our needs; the rest of the definition can be completed using any version of the conditional expectation. 


\section{C2. Proof of Proposition 2}

Assume that the uniform distribution is a mean-preserving spread of $\nu$ (or, equivalently, that $\nu$ is a mean-preserving contraction of the uniform distribution). As shown before the statement of the proposition, the uniform distribution is $1 / 2$ feasible. Hence, $P$ is $1 / 2$-feasible, by lemma 1 .

Conversely, let $\nu$ be a probability measure that is symmetric around $1 / 2$, and assume that $\nu$ is not a mean-preserving contraction of the uniform distribution. We show that $\nu \times \nu$ is not $1 / 2$-feasible.

Let $F(x)=\nu([0, x])$ be the cumulative distribution function of $\nu$. Since the uniform distribution is not a mean-preserving spread of $\nu$, there must be $y \in[0,1]$ such that

$$
H(y)=\int_{0}^{y} F(x) \mathrm{d} x-\int_{0}^{y} x \mathrm{~d} x=\int_{0}^{y} F(x) \mathrm{d} x-\frac{y^{2}}{2}>0 .
$$

Note that $H$ is continuous and furthermore differentiable. Since $\nu$ is symmetric and $\int_{0}^{1} F(x) \mathrm{d} x=1 / 2$, the function $H$ is also symmetric around $1 / 2: H(y)=$ $H(1-y)$.

The function $H$ vanishes at the end points of the interval and, as we noted above, is positive somewhere on $[0,1]$. Hence, it must have a global maximum $y \in(0,1 / 2]$. By construction, $H(y)>0$. Since $H$ is differentiable, we also have the first-order condition $H^{\prime}(y)=F(y)-y=0$.

Let $z=(1 / F(y)) \int_{0}^{y} x \mathrm{~d} \nu(x)$. We claim that $z<y / 2$. To see this, note that

$$
\begin{aligned}
z=\frac{1}{F(y)} \int_{0}^{y} x \mathrm{~d} F(x) & =\frac{1}{F(y)}\left(y F(y)-\int_{0}^{y} F(x) \mathrm{d} x\right) \\
& <y-\frac{y^{2}}{2 F(y)}=y-\frac{y}{2}=\frac{y}{2} .
\end{aligned}
$$

The second equality follows from integration by parts, the third equality holds because $\int_{0}^{y} F(x) \mathrm{d} x>y^{2} / 2$, and the forth inequality follows because $F(y)=y$.

Let $A_{1}=[1-y, 1]$ and $A_{2}=[0, y]$. Let $P=\nu \times \nu$. Note that, by symmetry, $\nu([y, 1])=1-F(y)=1-y$ and that, by independence, $P\left(A_{1} \times \overline{A_{2}}\right)=y(1-y)$. In addition, by construction, $\int_{A_{1}} x \mathrm{~d} P(x)=(1 / 2)-\int_{0}^{y} x \mathrm{~d} P(x)=(1 / 2)-y z$, and $\int_{A_{2}} x \mathrm{~d} P(x)=y z$. Therefore,

$$
\begin{aligned}
\int_{A_{1}} x \mathrm{~d} P(x)-\int_{A_{2}} x \mathrm{~d} P(x)-P\left(A_{1} \times \overline{A_{2}}\right) & =\frac{1}{2}-y z-y z-y(1-z) \\
& =\frac{1}{2}-2 y z-y+y^{2}>\frac{1}{2}-y \geq 0,
\end{aligned}
$$

where the second inequality follows because $z<1 / 2$. Therefore, theorem $2 \mathrm{im}-$ plies that $P=\nu \times \nu$ is not feasible. QED

\section{C3. Proof of Proposition 3}

Let $m \in(0,1)$ be the median of $\nu$. We first prove the proposition for the case where $\nu$ has no atom at $m$. We assume for simplicity that the number of agents is even; that is, $n=2 k$ (otherwise, we simply ignore the last agent). 
We consider the trading scheme $\left(a_{1}, \ldots, a_{2 k}\right)$ given by

$$
a_{i}(x)=-\mathbf{1}_{x<m}
$$

for $i \in\{1, \ldots, k\}$ and

$$
a_{i}(x)=-\mathbf{1}_{x>m}
$$

for $i \in\{k+1, \ldots, 2 k\}$. We argue that for large enough $n$ (i.e., large enough $k$ ) this trading scheme violates the condition of theorem 3 .

Let $B_{1} \sim \operatorname{Bin}(k, 1 / 2)$ denote the random variable of the number of agents $i=1, \ldots, k$ whose posterior is in $[0, m)$ (i.e., those who sell the product). Let $B_{2} \sim \operatorname{Bin}(k, 1 / 2)$ denote the random variable of the number of agents $i=$ $k+1, \ldots, 2 k$ whose posterior is in $(m, 1]$ (i.e., those who buy the product). Since $\nu^{n}$ is a product distribution, we have that $B_{1}$ is independent of $B_{2}$.

Note also that $\sum_{i=1}^{n} a_{i}\left(x_{i}\right)=B_{2}-B_{1}$, and so we have that the lower bound to the mediator's profit given by condition (8) equals

$$
k \int_{m}^{1} x \mathrm{~d} \nu(x)-k \int_{0}^{m} x \mathrm{~d} \nu(x)-\mathbb{E}\left(\max \left\{0, B_{2}-B_{1}\right\}\right) .
$$

We observe that $\mathbb{E}\left(\max \left\{0, B_{2}-B_{1}\right\}\right) \leq(k / 8)^{1 / 2}$; we have $\mathbb{E}\left(B_{2}-B_{1}\right)=0$ and $\mathbb{E}\left(\left(B_{2}-B_{1}\right)^{2}\right)=k / 2$. Jensen's inequality implies that $\mathbb{E}\left(\left|B_{2}-B_{1}\right|\right) \leq(k / 2)^{1 / 2}$, and by symmetry we deduce that $\mathbb{E}\left(\max \left\{B_{2}-B_{1}, 0\right\}\right) \leq(1 / 2)(k / 2)^{1 / 2}$.

It follows that for $k>(1 / 8)\left(\int_{m}^{1} x \mathrm{~d} \nu-\int_{0}^{m} x \mathrm{~d} \nu\right)^{-2}$, this lower bound on the mediator's profit is positive, and thus by theorem 3 the belief distribution $\nu^{n}$ is not feasible.

In case the distribution $\nu$ has an atom at $m$, the only needed change is to choose $a_{i}(m)$ so that the expected number of units that each agent buys or sells is $1 / 2$; the same calculation applies in this case. QED

\section{C4. Proof of Lemma 2}

Let $I_{0}=\left(\left(S_{i}\right)_{i \in N}, \mathbb{P}\right)$ be an information structure with prior $p$ that induces $P$. By the law of total expectation,

$$
x_{i}=\mathbb{P}\left(\omega=\mathrm{h} \mid s_{i}\right)=\mathbb{P}\left(\omega=\mathrm{h} \mid x_{i}\right)
$$

almost everywhere. Hence, if we define a new information structure $I^{\prime}$ for which the signals are $s_{i}^{\prime}=x_{i}$ and the beliefs are, accordingly, $x_{i}^{\prime}=\mathbb{P}\left(\omega=\mathrm{h} \mid s_{i}^{\prime}\right)$, we will have that

$$
x_{i}^{\prime}=s_{i}^{\prime},
$$

and so we have proved the second part of the claim.

Denote by $P^{\ell}$ and $P^{\mathrm{h}}$ the conditional distributions of $\left(x_{1}^{\prime}, \ldots, x_{n}^{\prime}\right)$, conditioned on $\omega=\ell$ and $\omega=\mathrm{h}$, respectively. Then, clearly, $P=(1-p) \cdot P^{\ell}+p \cdot P^{\mathrm{h}}$.

Finally, equation (A1) holds, since by Bayes's law and the fact that $x_{i}^{\prime}=s_{i}^{\prime}$,

$$
x_{i}^{\prime}=\mathbb{P}\left(\omega=\mathrm{h} \mid x_{i}^{\prime}\right)=p \cdot \frac{\mathrm{d} P_{i}^{\mathrm{h}}}{\mathrm{d} P_{i}}\left(x_{i}^{\prime}\right) .
$$




\section{C5. Proof of Proposition 4}

Let $B=\left(N, p,\left(A_{i}\right)_{i},\left(u_{i}\right)_{i}, u_{\mathrm{s}}\right)$ be a first-order Bayesian persuasion problem. By our equilibrium refinement assumption, for each receiver $i$ there is a map $\alpha_{i}:[0,1] \rightarrow A_{i}$ such that in equilibrium receiver $i$ chooses action $\tilde{a}_{i}=\alpha_{i}\left(x_{i}\right)$ when their posterior is $x_{i}$. Hence, if we set

$$
v\left(x_{1}, \ldots, x_{n}\right)=u_{\mathrm{s}}\left(\alpha_{1}\left(x_{1}\right), \ldots, \alpha_{n}\left(x_{n}\right)\right),
$$

then, given an information structure chosen by the sender,

$$
\mathbb{E}\left(u_{\mathrm{s}}\left(\tilde{a}_{1}, \ldots, \tilde{a}_{n}\right)\right)=\mathbb{E}\left(u_{\mathrm{s}}\left(\alpha_{1}\left(x_{1}\right), \ldots, \alpha_{n}\left(x_{n}\right)\right)\right)=\mathbb{E}\left(v\left(x_{1}, \ldots, x_{n}\right)\right) .
$$

Thus, if we denote by $P$ the posterior belief distribution induced by the chosen information structure, we have shown that the sender's expected utility is

$$
\int v\left(x_{1}, \ldots, x_{n}\right) \mathrm{d} P\left(x_{1}, \ldots, x_{n}\right) .
$$

Since the sender, by choosing the information structure, can choose any $p$-feasible distribution, we arrive at the desired conclusion. QED

\section{C6. Proof of Proposition 5}

We first show that every information structure $I=\left(S_{1}, S_{2}, \mathbb{P}\right)$ with prior $p$ chosen by the sender yields utility at most $(1-p) p$.

This proof uses the fact that conditional expectations are orthogonal projections in the Hilbert space of square-integrable random variables. We now review this fact.

For the probability space $\left(\Omega \times S_{1} \times S_{2}, \mathbb{P}\right)$, denote by $\mathcal{L}^{2}$ the Hilbert space of square-integrable random variables, equipped with the usual inner product $(X, Y)=\mathbb{E}(X \cdot Y)$ and corresponding norm $\|X\|=\left(\mathbb{E}\left(X^{2}\right)\right)^{1 / 2}$.

Given a sub-sigma-algebra $\mathcal{G} \subseteq \mathcal{F}$, denote by $\mathcal{L}^{2}(\mathcal{G}) \subseteq \mathcal{L}^{2}$ the closed subspace of $\mathcal{G}$-measurable, square-integrable random variables. Recall that the map $X \mapsto$ $E(X \mid \mathcal{G})$ is simply the orthogonal projection $\mathcal{L}^{2} \rightarrow \mathcal{L}^{2}(\mathcal{G})$.

The following is an elementary lemma regarding Hilbert spaces. We will, of course, apply it to $\mathcal{L}^{2}$.

Lemma 5. Let $u$ be a vector in a Hilbert space $U$, and let $w$ be an orthogonal projection of $u$ to a closed subspace $W \subseteq U$. Then, $w$ lies on a sphere of radius $(1 / 2)\|u\|$ around $(1 / 2) u$, that is, $\|w-(1 / 2) u\|=(1 / 2)\|u\|$.

Proof. Since $w$ is an orthogonal projection of $u$, we can write $u=w+w^{\prime}$, where $w$ and $w^{\prime}$ are orthogonal. This orthogonality implies that $\left\|w-w^{\prime}\right\|=$ $\left\|w+w^{\prime}\right\|=\|u\|$. It follows that

$$
\left\|w-\frac{1}{2} u\right\|=\left\|w-\frac{1}{2}\left(w+w^{\prime}\right)\right\|=\left\|\frac{1}{2} w-\frac{1}{2} w^{\prime}\right\|=\frac{1}{2}\left\|w-w^{\prime}\right\|=\frac{1}{2}\|u\| .
$$

QED

Let $X=\mathbf{1}_{\omega=\mathrm{h}}$ be the indicator of the event that the state is high. Hence, $x_{i}=\mathbb{E}\left(X \mid \mathcal{F}_{i}\right)$, where $\mathcal{F}_{i}=\sigma\left(s_{i}\right)$ is the sigma-algebra generated by the private signal $s_{i}$. Denote $\hat{X}=X / p-1$. Hence, $\mathbb{P}(\hat{X}=1 / p-1)=p, \mathbb{P}(\hat{X}=-1)=$ $1-p$, and $\|\hat{X}\|^{2}=(1-p) / p$. Denote $\hat{X}_{i}=x_{i} / p-1$, so that $\hat{X}_{i}=\mathbb{E}\left(\hat{X} \mid \mathcal{F}_{i}\right)$. 
Since $\hat{X}_{i}$ is the projection of $\hat{X}$ to the subspace $\mathcal{L}^{2}\left(\mathcal{F}_{i}\right)$, it follows from lemma 5 that

$$
\left\|\hat{X}_{i}-\frac{\hat{X}}{2}\right\|=\frac{1}{2} \sqrt{\frac{1-p}{p}} .
$$

Therefore, by the triangle inequality, $\left\|\hat{X}_{1}-\hat{X}_{2}\right\| \leq[(1-p) / p]^{1 / 2}$, and since $\hat{X}_{i}=$ $x_{i} / p-1$, we get that $\left\|x_{1} / p-x_{2} / p\right\| \leq[(1-p) / p]^{1 / 2}$, or

$$
\mathbb{E}\left(\left(\frac{x_{1}}{p}-\frac{x_{2}}{p}\right)^{2}\right) \leq \frac{1-p}{p} .
$$

Thus,

$$
\mathbb{E}\left(\left(x_{1}-x_{2}\right)^{2}\right) \leq(1-p) p .
$$

To finish the proof of proposition 5 , we note that a simple calculation shows that the sender can achieve the expected utility $(1-p) p$ by completely informing one agent and giving no information to the other. That is, by inducing the joint posterior distribution,

$$
P=p \cdot \delta_{1, p}+(1-p) \delta_{0, p} .
$$

QED

\section{C7. Remark on the Polarizing First-Order Bayesian Persuasion Problem}

with $a<2$ and $p=1 / 2$

For the first-order Bayesian persuasion problem $B_{a}$ given by $v\left(x_{1}, x_{2}\right)=\left|x_{1}-x_{2}\right|^{a}$ and symmetric prior $p=1 / 2$, we argue that $P=(1 / 2) \delta_{1,1 / 2}+(1 / 2) \delta_{0,1 / 2}$ is the optimal posterior distribution for all $a \in(0,2)$; thus, the value $V\left(B_{a}\right)$ of the problem equals $1 / 2^{a}$. To prove this, it is enough to check that the upper bound $V\left(B_{a}\right) \leq 1 / 2^{a}$.

Consider an arbitrary $1 / 2$-feasible policy $P^{\prime}$. Hölder's inequality implies

$$
\int v\left(x_{1}, x_{2}\right) \mathrm{d} P^{\prime}=\int v\left(x_{1}, x_{2}\right) \cdot 1 \mathrm{~d} P^{\prime} \leq\left(\int\left(v\left(x_{1}, x_{2}\right)\right)^{q} \mathrm{~d} P^{\prime}\right)^{1 / q} \cdot\left(\int 1^{q^{\prime}} \mathrm{d} P^{\prime}\right)^{1 / q^{\prime}},
$$

where $q, q^{\prime}>0$ and $(1 / q)+\left(1 / q^{\prime}\right)=1$. Picking $q=2 / a$ and taking the supremum over $P^{\prime}$ on both sides, we get $V\left(B_{a}\right) \leq\left(V\left(B_{2}\right)\right)^{a / 2}$. By proposition 5 , $V\left(B_{2}\right)=1 / 4$, and we obtain the required upper bound.

\section{C8. Proof of Proposition 6}

It is immediate to check that the expected utility for the distribution $P$ given in the theorem statement is $1 / 32$. It thus remains to prove that no other distribution can achieve a higher expected utility.

We retain the notation used in the proof of proposition 4 . Let $\left(x_{1}, x_{2}\right)$ be the posterior beliefs of two agents induced by some information structure. Since the prior is $p=1 / 2, \hat{X}_{i}=2 x_{i}-1$, and so

$$
\mathbb{E}\left(v\left(x_{1}, x_{2}\right)\right)=-\mathbb{E}\left(\left(x_{1}-1 / 2\right) \cdot\left(x_{2}-1 / 2\right)\right)=-\frac{1}{4} \mathbb{E}\left(\hat{X}_{1} \cdot \hat{X}_{2}\right) .
$$


We recall that $(\cdot, \cdot)$ denotes the inner product in the Hilbert space $\mathcal{L}^{2}$. Thus, we can write

$$
\mathbb{E}\left(v\left(x_{1}, x_{2}\right)\right)=-\frac{1}{4}\left(\hat{X}_{1}, \hat{X}_{2}\right) .
$$

By lemma 5, the vectors $\hat{X}_{1}, \hat{X}_{2} \in \mathcal{L}^{2}$ lie on a sphere of radius $(1 / 2)\|\hat{X}\|$ around $(1 / 2) \hat{X} \in \mathcal{L}^{2}$. Note that since $p=1 / 2,\|\hat{X}\|=1$. Since $\left\{\hat{X}, \hat{X}_{1}, \hat{X}_{2}\right\}$ span a subspace that is (at most) three-dimensional, the question is reduced to the following elementary question in three-dimensional geometry: given a vector $w \in \mathbb{R}^{3}$, what is the minimum of the inner product $\left(u_{1}, u_{2}\right)$ as $u_{1}, u_{2} \in \mathbb{R}^{3}$ range over the sphere of radius $\|w\|$ around $w$ ? Lemma 6 states that the minimum is $-(1 / 2)\|w\|^{2}$. Hence, by equation (C5),

$$
\mathbb{E}\left(v\left(x_{1}, x_{2}\right)\right) \leq \frac{1}{32}
$$

QED

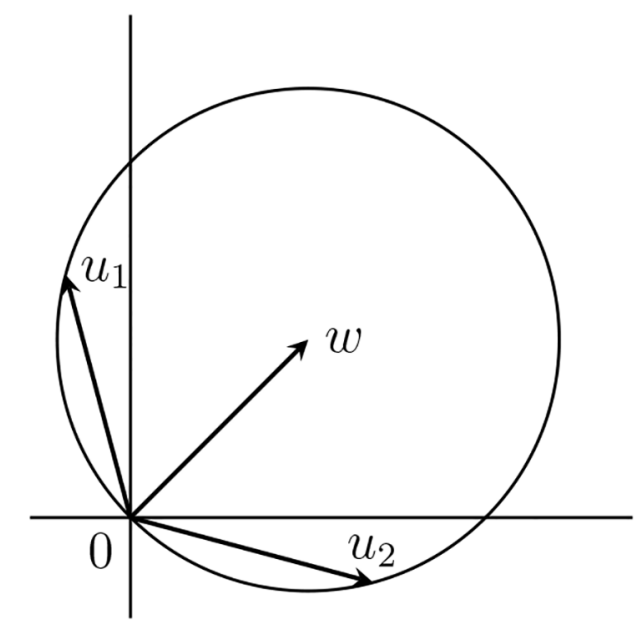

FIG. C1.-Lemma 6 states that $-1 / 2$ is the smallest possible inner product between two vectors that lie on a unit sphere that intersects the origin. The depicted $u_{1}$ and $u_{2}$ achieve this minimum.

Lemma 6. Let $w \in \mathbb{R}^{3}$. Then,

$$
\min \left\{\left(u_{1}, u_{2}\right):\left\|w-u_{1}\right\|=\left\|w-u_{2}\right\|=\|w\|\right\}=-\frac{1}{2}\|w\|^{2} .
$$

The proof is left as an exercise to the reader. Alternatively, this problem can be solved symbolically using the Mathematica command

Minimize $[\{x 1 * y 1+x 2 * y 2+x 3 * y 3$,

$\left.(x 1-1)^{\wedge} 2+(x 2)^{\wedge} 2+(x 3)^{\wedge} 2==1 \& \&(y 1-1)^{\wedge} 2+(y 2)^{\wedge} 2+(y 3)^{\wedge} 2==1\right\}$,

$\{x 1, x 2, x 3, y 1, y 2, y 3\}]$. 


\section{C9. Proof of Theorem 4}

We start by constructing an extreme point of $\mathcal{P}_{p}^{2}$ with infinite support. Thereafter, we extend this construction for $N \geq 2$ receivers.

Consider the following information structure. Let $S_{1}=S_{2}=\{1,2, \ldots\}$. Choose at random a number $K$, which is distributed geometrically with parameter $r$ that depends on the state: when $\omega=\ell, r=2 / 3$, and when $\omega=\mathrm{h}, r=1 / 2$. The signals $\left(s_{1}, s_{2}\right)$ that the agents receive are equal to $(K, K)$ when $\omega=\ell$ and to $(K+1, K)$ when $\omega=\mathrm{h}$.

A simple calculation shows that when an agent 1 observes a signal $s_{1}=k$, her posterior $x_{1}(k)=t_{k}$ and that when agent 2 observes $s_{2}=k$, her posterior is $x_{2}(k)=w_{k}$, where $t_{k}$ and $w_{k}$ are given by

$$
\begin{aligned}
& t_{k}=\left\{\begin{array}{cc}
\frac{2^{-k+1} p}{2^{-k+1} p+2 \cdot 3^{-k}(1-p)}, & k \geq 2, \\
0, & k=1,
\end{array}\right. \\
& w_{k}=\frac{2^{-k} p}{2^{-k} p+2 \cdot 3^{-k}(1-p)}, \quad k \geq 1 .
\end{aligned}
$$

The induced conditional distributions of $\left(x_{1}, x_{2}\right)$ are

$$
\begin{aligned}
& P^{\ell}=\sum_{k=1}^{\infty} 2 \cdot 3^{-k} \cdot \delta_{\left(t_{k}, w_{k}\right)}, \\
& P^{\mathrm{h}}=\sum_{k=1}^{\infty} 2^{-k} \cdot \delta_{\left(t_{k+1}, w_{k}\right)} .
\end{aligned}
$$

Note that $P^{\ell}$ and $P^{\text {h }}$ have disjoint supports; see figure 2.

Hence, the induced (unconditional) distribution is $P=(1-p) \cdot P^{\ell}+p \cdot P^{\mathrm{h}}$. It satisfies the pair of identities

$$
\begin{gathered}
t_{k} \cdot P\left(\left\{\left(t_{k}, w_{k}\right)\right\}\right)=\left(1-t_{k}\right) \cdot P\left(\left\{\left(t_{k}, w_{k-1}\right)\right\}\right), \quad k \geq 2, \\
w_{k} \cdot P\left(\left\{\left(t_{k}, w_{k}\right)\right\}\right)=\left(1-w_{k}\right) \cdot P\left(\left\{\left(t_{k+1}, w_{k}\right)\right\}\right), \quad k \geq 1,
\end{gathered}
$$

which we use below.

We first argue that $\left(P^{\ell}, P^{\mathrm{h}}\right)$ is the unique implementation of $P^{22}$

By lemma 2, for any pair $\left(Q^{\ell}, Q^{\mathrm{h}}\right)$ implementing a feasible distribution $Q$ the marginals satisfy $\mathrm{d} Q_{i}^{\mathrm{h}}(x)=(x / p) \mathrm{d} Q_{i}(x)$ for each agent $i \in N$. Since $Q^{\ell}=$ $\left(Q-p \cdot Q^{\mathrm{h}}\right) /(1-p)$, we get the complementary equation $\mathrm{d} Q_{i}^{\ell}(x)=[(1-x) /$ $(1-p)] \mathrm{d} Q_{i}(x)$. Combining the two equations, we obtain

$$
\frac{x}{p} \cdot \mathrm{d} Q_{i}^{\ell}(x)=\frac{1-x}{1-p} \cdot \mathrm{d} Q_{i}^{\mathrm{h}}(x) \quad \text { for } i \in N .
$$

Let $\left(\hat{P}^{\ell}, \hat{P}^{\mathrm{h}}\right)$ be an implementation of $P$. The identity (C8), applied to $i=1$ and $x=t_{k}$, gives the equation

${ }^{22}$ See sec. VI for the definition of an implementation as well as an example that shows that feasible distributions may have multiple implementations. 


$$
\frac{t_{1}}{p} \hat{P}^{\ell}\left(\left\{\left(t_{1}, w_{1}\right)\right\}\right)=\frac{1-t_{1}}{1-p} \hat{P}^{\mathrm{h}}\left(\left\{\left(t_{1}, w_{1}\right)\right\}\right)
$$

for $k=1$ and the following family of equations for $k \geq 2$ :

$$
\begin{aligned}
& \frac{t_{k}}{p}\left(\hat{P}^{\ell}\left(\left\{\left(t_{k}, w_{k}\right)\right\}\right)+\hat{P}^{\ell}\left(\left\{\left(t_{k}, w_{k-1}\right)\right\}\right)\right) \\
& =\frac{1-t_{k}}{1-p}\left(\hat{P}^{\mathrm{h}}\left(\left\{\left(t_{k}, w_{k}\right)\right\}\right)+\hat{P}^{\mathrm{h}}\left(\left\{\left(t_{k}, w_{k-1}\right)\right\}\right)\right) .
\end{aligned}
$$

Similarly, for $i=2$ and $x_{2}=w_{k}$, we get

$$
\begin{aligned}
& \frac{w_{k}}{p}\left(\hat{P}^{\ell}\left(\left\{\left(t_{k}, w_{k}\right)\right\}\right)+\hat{P}^{\ell}\left(\left\{\left(t_{k+1}, w_{k}\right)\right\}\right)\right) \\
& =\frac{1-w_{k}}{1-p}\left(\hat{P}^{\mathrm{h}}\left(\left\{\left(t_{k}, w_{k}\right)\right\}\right)+\hat{P}^{\mathrm{h}}\left(\left\{\left(t_{k+1}, w_{k}\right)\right\}\right)\right) .
\end{aligned}
$$

We now show that these equations and the condition $P=(1-p) \hat{P}^{\ell}+p \cdot \hat{P}^{\mathrm{h}}$ completely determine the pair $\left(\hat{P}^{\ell}, \hat{P}^{\mathrm{h}}\right)$. Since $t_{1}=0$, equation $(\mathrm{C} 9)$ results in $\hat{P}^{\mathrm{h}}\left(\left\{\left(t_{1}, w_{1}\right)\right\}\right)=0$, and hence the entire mass $[1 /(1-p)] P\left(\left\{\left(t_{1}, w_{1}\right)\right\}\right)$ of the point $\left(t_{1}, w_{1}\right)$ must be assigned to $\hat{P}^{\ell}$. Given this, the equality (C11) implies that the entire mass $(1 / p) P\left(\left\{\left(t_{2}, w_{1}\right)\right\}\right)$ of the point $\left(t_{2}, w_{1}\right)$ must be assigned to $\hat{P}^{\mathrm{h}}$. Indeed, expressing $\hat{P}^{\ell}\left(\left\{\left(t_{k}, w_{k}\right)\right\}\right)$ and $\hat{P}^{\mathrm{h}}\left(\left\{\left(t_{k+1}, w_{k}\right)\right\}\right)$ through $P$ and taking into account that $\hat{P}^{\mathrm{h}}\left(\left\{\left(t_{k}, w_{k}\right)\right\}\right)=0$ for $k=1$, we rewrite equation $(\mathrm{C} 11)$ as

$$
\begin{aligned}
& \frac{w_{k}}{p}\left(\frac{1}{1-p} P\left(\left\{\left(t_{k}, w_{k}\right)\right\}\right)+\hat{P}^{\ell}\left(\left\{\left(t_{k+1}, w_{k}\right)\right\}\right)\right) \\
& =\frac{1-w_{k}}{1-p}\left(\frac{P\left(\left\{\left(t_{k+1}, w_{k}\right)\right\}\right)-(1-p) \hat{P}^{\ell}\left(\left\{\left(t_{k+1}, w_{k}\right)\right\}\right)}{p}\right)
\end{aligned}
$$

for $k=1$. This equality and the identity (C7) lead to $\hat{P}^{\ell}\left(\left\{\left(t_{2}, w_{1}\right)\right\}\right)=0$.

Next, a similar argument demonstrates that the entire mass $[1 /(1-p)] P\left(\left\{\left(t_{2}, w_{2}\right)\right\}\right)$ of the point $\left(t_{2}, w_{2}\right)$ must be assigned to $\hat{P}^{\ell}$. Indeed, we know that $\hat{P}^{\ell}\left(\left\{\left(t_{k}, w_{k-1}\right)\right\}\right)=0$ for $k=2$, which allows us to rewrite equation $(\mathrm{C} 10)$ as

$$
\begin{aligned}
& \frac{t_{k}}{p}\left(\frac{P\left(\left\{\left(t_{k}, w_{k}\right)\right\}-p \cdot \hat{P}^{\mathrm{h}}\left(\left\{\left(t_{k}, w_{k}\right)\right\}\right)\right)}{1-p}\right) \\
& =\frac{1-t_{k}}{1-p}\left(\hat{P}^{\mathrm{h}}\left(\left\{\left(t_{k}, w_{k}\right)\right\}\right)+\frac{1}{p} P\left(\left\{\left(t_{k}, w_{k-1}\right)\right\}\right)\right)
\end{aligned}
$$

for $k=2$. Combining this equality with identity $(\mathrm{C} 6)$, we get $\hat{P}^{\mathrm{h}}\left(\left\{\left(t_{2}, w_{2}\right)\right\}\right)=0$. We proceed inductively: knowing that $\hat{P}^{\mathrm{h}}\left(\left\{\left(t_{k}, w_{k}\right)\right\}\right)=0$, we deduce equality (C12) and infer, using equation (C7), that $\hat{P}^{\ell}\left(\left\{\left(t_{k+1}, w_{k}\right)\right\}\right)=0$; from this, we derive 
equality (C13) and, with the help of equation (C6), see that $\hat{P}^{\mathrm{h}}\left(\left\{\left(t_{k+1}, w_{k+1}\right)\right\}\right)=0$, and so on. Thus, $\hat{P}^{\ell}$ coincides with $[1 /(1-p)] P$ restricted to $\left\{\left(t_{k}, w_{k}\right): k \geq 1\right\}$, and $\hat{P}^{\mathrm{h}}$ coincides with $(1 / p) P$ restricted to $\left\{\left(t_{k+1}, w_{k}\right): k \geq 1\right\}$, and so $\hat{P}^{\ell}=P^{\ell}$ and $\hat{P}^{\mathrm{h}}=$ $P^{\mathrm{h}}$. Hence, $P$ has a unique implementation.

Given a convex combination $P=\alpha Q+(1-\alpha) \hat{Q}$, where $Q$ and $\hat{Q}$ are both feasible and $\alpha \in(0,1)$, our goal is to show that $Q=P$. Let $\left(Q^{\ell}, Q^{\mathrm{h}}\right)$ and $\left(\hat{Q}^{\ell}, \hat{Q}^{\mathrm{h}}\right)$ be some pairs of conditional probability distributions that implement $Q$ and $\hat{Q}$, respectively. The pair $\left(\alpha Q^{\ell}+(1-\alpha) \hat{Q}^{\ell}, \alpha Q^{\mathrm{h}}+(1-\alpha) \hat{Q}^{\mathrm{h}}\right)$ implements $P$. From the uniqueness of $P$ 's implementation, we deduce that $\operatorname{supp}\left(Q^{\ell}\right) \subset \operatorname{supp}\left(P^{\ell}\right)$ and $\operatorname{supp}\left(Q^{\mathrm{h}}\right) \subset \operatorname{supp}\left(P^{\mathrm{h}}\right)$ and thus that $Q^{\ell}\left(\left\{\left(t_{k+1}, w_{k}\right)\right\}\right)=Q^{\mathrm{h}}\left(\left\{\left(t_{k}, w_{k}\right)\right\}\right)=0$. Therefore, condition (C8) implies

$$
\begin{gathered}
\frac{t_{k}}{p} \cdot Q^{\ell}\left(\left\{\left(t_{k}, w_{k}\right)\right\}\right)=\frac{1-t_{k}}{1-p} \cdot Q^{\mathrm{h}}\left(\left\{\left(t_{k}, w_{k-1}\right)\right\}\right), \quad k \geq 2, \\
\frac{w_{k}}{p} \cdot Q^{\ell}\left(\left\{\left(t_{k}, w_{k}\right)\right\}\right)=\frac{1-w_{k}}{1-p} \cdot Q^{\mathrm{h}}\left(\left\{\left(t_{k+1}, w_{k}\right)\right\}\right), \quad k \geq 1 .
\end{gathered}
$$

This family of equations uniquely determines the weights of each point $\left(t_{k}, w_{k}\right)$ and $\left(t_{k+1}, w_{k}\right), k \geq 1$, up to a multiplicative factor, which is pinned down by the condition that $Q$ is a probability measure. Hence, in the decomposition $P=$ $\alpha Q+(1-\alpha) \hat{Q}$, the distribution $Q$ is unique. Thus, $Q$ must be equal to $P$, and hence $P$ is an extreme point.

This construction extends to $N \geq 2$, by sending no information to the remaining $N-2$ agents. The resulting distribution over posteriors is an extreme point because of the same arguments.

Now we check the singularity of the extreme points of $\mathcal{P}_{p}^{N}$ with respect to the Lebesgue measure $\lambda$ on the unit cube $[0,1]^{N}$. The proof relies on the classical theorem of Lindenstrauss (1965), which states that all extreme points of the set of all probability measures with given marginals are singular. Lindenstrauss (1965) proved this result for $n=2$, and Shortt (1986) extended it to general $n$. In appendix E (theorem 5), we include an alternative proof for $n \geq 2$, closely following the original proof of Lindenstrauss. Here, we show how to apply this result to our problem.

By lemma 2, any $p$-feasible distribution $P$ can be represented as $(1-p) P^{\ell}+$ $p \cdot P^{\mathrm{h}}$, where the marginals of the pair $\left(P^{\ell}, P^{\mathrm{h}}\right)$ satisfy the identity

$$
\frac{x}{p} d P_{i}^{\ell}(x)=\left(1-\frac{x}{p}\right) d P_{i}^{\mathrm{h}}(x) .
$$

Denote by $\mathcal{H}_{p}^{N}$ the set of all pairs satisfying equation (C14). The set of $p$-feasible distributions $\mathcal{P}_{p}^{N}$ is, therefore, the image of the convex set $\mathcal{H}_{p}^{N}$ under the linear $\operatorname{map}\left(P^{\ell}, P^{\mathrm{h}}\right) \rightarrow(1-p) P^{\ell}+p \cdot P^{\mathrm{h}}$. The extreme points of $\mathcal{P}_{p}^{N}$ are hence all contained in the image of the extreme points of $\mathcal{H}_{p}^{N}$.

So it is enough to check that in any extreme pair $\left(P^{\ell}, P^{\mathrm{h}}\right)$, both measures are necessarily singular with respect to $\lambda$. We check singularity of $P^{\ell}$; the argument for $P^{\mathrm{h}}$ is analogous. We assume toward a contradiction that $P^{\ell}$ is not singular. Then, by theorem $5, P^{\ell}$ is not an extreme point among measures with the same marginals and thus can be represented as $P^{\ell}=(1 / 2) Q+(1 / 2) Q^{\prime}$, where $Q$ and 
$Q^{\prime}$ have the same marginals as $P^{\ell}$ and $Q \neq Q^{\prime}$. This induces the representation of $\left(P^{\ell}, P^{\mathrm{h}}\right)$ as the average of $\left(Q, P^{\mathrm{h}}\right)$ and $\left(Q^{\prime}, P^{\mathrm{h}}\right)$, where both pairs satisfy equation (C14). A contradiction results. QED

Proposition 8. The set of $p$-feasible posterior distributions is a convex, weak $^{*}$ compact subset of $\Delta\left([0,1]^{n}\right)$.

Proof. Pick a pair of feasible distributions $P, P^{\prime} \in \mathcal{P}_{p}^{N}$, and consider the corresponding $Q, Q^{\prime} \in \Delta\left([0,1]^{N}\right)$ that satisfy the conditions of lemma 3 for $P$ and $P^{\prime}$, respectively. For a convex combination $P^{\prime \prime}=\alpha P+(1-\alpha) P^{\prime}$, the conditions of the lemma are satisfied by $Q^{\prime \prime}=\alpha Q+(1-\alpha) Q^{\prime}$. Thus, $P^{\prime \prime}$ also belongs to $\mathcal{P}_{p}^{N}$, and so we have shown that the set of feasible distributions is convex.

To verify the weak ${ }^{*}$ compactness of $\mathcal{P}_{p}^{N}$, consider a sequence of $p$-feasible distributions $P^{(k)}$ weakly converging to some $P^{(\infty)} \in \Delta\left([0,1]^{N}\right)$ as $k \rightarrow \infty$. To prove weak ${ }^{*}$ compactness, we show that the limit distribution $P^{(\infty)}$ is also feasible. For each $P^{(k)}$, select some $Q^{(k)}$ from lemma 3 . The set of all probability distributions on $[0,1]^{N}$ is weak ${ }^{*}$ compact, and therefore there is a subsequence $Q^{\left(k_{m}\right)}$ weakly converging to some $Q^{(\infty)} \in \Delta\left([0,1]^{N}\right)$.

The conditions of lemma 3 can be rewritten in an equivalent integrated form. Condition 1 of the lemma becomes

$$
\int f\left(x_{1}, \ldots, x_{n}\right)\left(\frac{1}{p} \mathrm{~d} P\left(x_{1}, \ldots, x_{n}\right)-\mathrm{d} Q\left(x_{1}, \ldots, x_{n}\right)\right) \geq 0
$$

for any nonnegative continuous function $f$ on the unit cube. Condition 2 of the lemma is equivalent to

$$
\int g\left(x_{i}\right)\left(\frac{x_{i}}{p} \mathrm{~d} P\left(x_{1}, \ldots, x_{n}\right)-\mathrm{d} Q\left(x_{1}, \ldots, x_{n}\right)\right)=0
$$

for any agent $i \in N$ and any continuous $g$ of arbitrary sign on the unit interval.

With this reformulation, it is immediate that both conditions are closed and hence withstand the weak ${ }^{*}$ limit. Therefore, since $P^{\left(k_{m}\right)}, Q^{\left(k_{n}\right)}$ satisfy the conditions (C15) and (C16), the limiting pair $P^{(\infty)}, Q^{(\infty)}$ satisfies them as well. We deduce that $P^{(\infty)}$ is feasible. QED

\section{Appendix D}

\section{Checking on Intervals Is Not Sufficient}

In this appendix, we show that restricting condition (6) to intervals $A_{1}, A_{2}$ provides a condition that - while clearly necessary — is not sufficient for feasibility.

To see this, consider the following distribution $P$, depicted in figure D1. It is parameterized by small $\varepsilon>0$ and is supported on four points: two "heavy" points, $(1 / 4-\varepsilon / 4,1 / 2)$ and $(3 / 4,1 / 2)$ with probabilities $(1-\varepsilon) / 2$ and $1 / 2$, respectively, and two "light" points, $(1 / 2-\varepsilon / 4,0)$ and $(1 / 2-\varepsilon / 4,1)$, each with probability $\varepsilon / 4$. Thus,

$$
P=\frac{1-\varepsilon}{2} \delta_{(1-\varepsilon) / 4,1 / 2}+\frac{1}{2} \delta_{3 / 4,1 / 2}+\frac{\varepsilon}{4}\left(\delta_{1 / 2-\varepsilon / 4,0}+\delta_{1 / 2-\varepsilon / 4,1}\right) .
$$




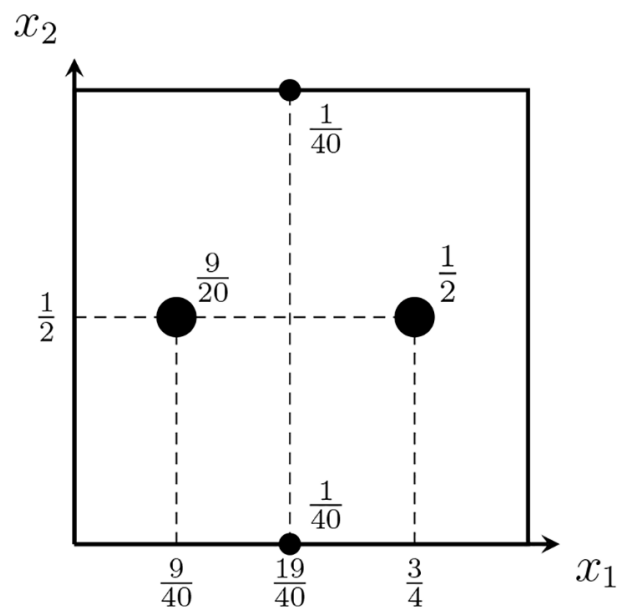

FIG. D1.-The distribution defined in equation (D1), for $\varepsilon=1 / 10$. Larger circles indicate the "heavy" points and smaller circles the "light" points.

This distribution satisfies the martingale condition with prior $1 / 2$; however, it is not $1 / 2$-feasible. To see that, pick $A_{1}=\{(1-\varepsilon) / 4,3 / 4\}$ and $A_{2}=\{1 / 2\}$; then, $A_{1} \times \overline{A_{2}}$ has zero $P$-measure and condition (6) is violated:

$$
0 \geq \frac{1-\varepsilon}{4} \cdot \frac{1-\varepsilon}{2}+\frac{3}{4} \cdot \frac{1}{2}-\frac{1}{2}\left(\frac{1-\varepsilon}{2}+\frac{1}{2}\right)=\frac{\varepsilon^{2}}{8} .
$$

We now check that none of the inequalities in condition (6) is violated for intervals $A_{1}, A_{2}$. Since $P$ satisfies the martingale condition, it suffices to check the left-hand inequality, ${ }^{23}$

$$
P\left(A_{1} \times \overline{A_{2}}\right) \geq \int_{A_{1}} x \mathrm{~d} P_{1}(x)-\int_{A_{2}} x \mathrm{~d} P_{2}(x) .
$$

Since $P$ has finite support, different choices of $A_{1}, A_{2}$ yield the same inequality if each set contains the same points of the support of $P_{1}$ and $P_{2}$, respectively. Thus, we need to check that condition (D2) is satisfied if $A_{1}$ is a subset of $\{(1-$ $\varepsilon) / 4,1 / 2-\varepsilon / 4,3 / 4\}$ and $A_{2}$ a subset of $\{0,1 / 2,1\}$, except the cases of $A_{1}=$ $\{(1-\varepsilon) / 4,3 / 4\}$ and $A_{2}=\{0,1\}$, which exclude the middle points and do not correspond to any interval.

We consider the following cases:

- Inequality (D2) holds if one of the sets $A_{i}$ is empty or contains the whole support of $P_{i}$, as it then boils down to the martingale condition, which we have already verified.

- Consider the case when $A_{1} \times \overline{A_{2}}$ contains exactly one heavy point; by the interval constraint, if it contains two, then $A_{1}$ contains the support of $P_{1}$, which

${ }^{23}$ Using the martingale condition $\int_{A_{1}} x \mathrm{~d} P_{1}(x)+\int_{A_{1}} x \mathrm{~d} P_{1}(x)=p$, the right-hand inequality in condition (6) follows from the left-hand one by a simple calculation. 
is the case we already considered. In this case, $P\left(A_{1} \times \overline{A_{2}}\right) \geq(1-\varepsilon) / 2$. On the other hand, the integral $\int_{A_{1}} x \mathrm{~d} P_{1}(x)$ does not exceed $1 / 2-(3 / 4)$. $(1 / 2)=1 / 8$ if $A_{1}$ excludes the rightmost heavy point and it does not exceed $1 / 2-[(1-\varepsilon) / 4][(1-\varepsilon) / 2]$. We see that for $\varepsilon$ small enough (e.g., $\varepsilon=1 / 10)$, condition (D2) is satisfied regardless of the choice of $A_{2}$, since $(1-\varepsilon) /$ $2 \geq \max \{1 / 8,1 / 2-[(1-\varepsilon) / 4][(1-\varepsilon) / 2]\}$.

- Consider the remaining case, in which there are no heavy points in $A_{1} \times \overline{A_{2}}$ and both $A_{1}$ and $A_{2}$ are nonempty strict subsets of the supports. This can be possible only if $A_{2}$ contains $1 / 2$ or $A_{1}=\{1 / 2-\varepsilon / 4\}$. In the former case, $\int_{A_{2}} x \mathrm{~d} P_{2}(x) \geq 1 / 2-\varepsilon / 4$, which for small $\varepsilon$ (1/10 suffices) exceeds $\int_{A_{1}} x \mathrm{~d} P_{1}(x)$ for any $A_{1}$ excluding at least one of the heavy points (see the bounds above). Hence, the right-hand side of condition (D2) is negative, and the inequality is satisfied. Consider the remaining case of $A_{1}=\{1 / 2-\varepsilon / 4\}$ and $A_{2}$ either $\{0\}$ or $\{1\}$. The left-hand side of condition (D2) equals $\varepsilon / 4$, and $\int_{A_{1}} x \mathrm{dP}_{1}(x)=(1 / 2-\varepsilon / 4) \cdot \varepsilon / 2 \leq \varepsilon / 4$. Thus, condition (D2) is satisfied on all intervals.

This example also demonstrates that the condition of Ziegler (2020, theorem 1) is not sufficient for feasibility. Using our notation, his condition can be written as follows:

$$
\begin{gathered}
\max \left\{\int_{0}^{a} x \mathrm{~d} P_{1}(x)+\int_{0}^{b} x \mathrm{~d} P_{2}(x)-p, \int_{0}^{a}(1-x) \mathrm{d} P_{1}(x)+\int_{0}^{b}(1-x) \mathrm{d} P_{2}(x)-(1-p)\right\} \\
\leq P([0, a] \times[0, b]) \\
\leq \min \left\{\int_{0}^{a}(1-x) \mathrm{d} P_{1}(x)+\int_{0}^{b} x \mathrm{~d} P_{2}(x), \int_{0}^{a} x \mathrm{~d} P_{1}(x)+\int_{0}^{b}(1-x) \mathrm{d} P_{2}(x)\right\}
\end{gathered}
$$

for every $a, b \in[0,1]$. Simple computations show that, for every given $a, b \in[0,1]$, this condition is equivalent to four of our conditions (see eq. [6]) for all possible combinations of $A_{1}=[0, a]$ or $A_{1}=[a, 1]$ and $A_{2}=[0, b]$ or $A_{2}=[b, 1]$.

The condition for feasibility in Ziegler (2020) is essentially the same as our condition (6), but restricted to interval subsets $A_{1}, A_{2} \subset[0,1]$, where each interval has a boundary point either at 0 or at 1 . If the marginals $P_{1}$ and $P_{2}$ are supported on at most two points, such intervals exhaust all possible nontrivial sets in equation (6); in this case, Ziegler's condition is necessary and sufficient for feasibility. However, the example above demonstrates that this condition becomes insufficient if the support contains at least three points.

\section{Appendix E}

\section{Singularity of Extreme Measures with Given Marginals}

In this appendix, we formulate an extension of a celebrated result of Lindenstrauss (1965) regarding extreme points of the set of measures with given marginals. The extension is twofold: the classic result assumes $n=2$ and uniform marginals, and we get rid of both assumptions. This extension can also be deduced from a more general statement by Shortt (1986), which allows for nonorthogonal multidimensional projections. 
For the reader's convenience, we include a proof similar to Lindenstrauss's.

Theorem 5. ( $n=2$ [Lindenstrauss 1965]; $n \geq 2$ [Shortt 1986]). Any extreme point $P$ of the set of probability measures on $[0,1]^{N}$ with given one-dimensional marginals $\nu_{i} \in \Delta([0,1]), i \in N$, is singular with respect to the Lebesgue measure $\lambda$ on the unit cube. In other words, for each extreme $P$, there exists a measurable set $B$ such that $P(B)=1$ and $\lambda(B)=0$.

Proof. Assume the converse: $P$ is not singular. By the Lebesgue decomposition theorem, the continuous part of $P$ can be singled out: $P=\mu+\lambda^{\perp}$, where $\mu \neq 0$ is absolutely continuous with respect to $\lambda(\mathrm{d} \mu=f \mathrm{~d} \lambda$, with a nonnegative integrable density $f), \lambda^{\perp}$ is singular with respect to $\lambda$.

Consider the Lebesgue space $\mathcal{L}^{1}(\mu)$ of integrable functions with respect to $\mu$ (defined $\mu$-everywhere) and its closed subspace $\mathcal{S}$ generated by "separable" 24 functions $g\left(x_{1}, \ldots, x_{n}\right)=\sum_{i \in N} g_{i}\left(x_{i}\right)$, where $g_{i} \in \mathcal{L}^{1}(\mu), i \in N$, depends on the variable $x_{i}$ only.

Let us assume that $\mathcal{S} \neq \mathcal{L}^{1}(\mu)$, that is, that separable integrable functions are not dense in all integrables (we check this condition at the end of the proof). Now we show that under this assumption, $\mu$ can be represented as a convex combination of $(1 / 2) \mu^{\prime}+(1 / 2) \mu^{\prime \prime}$, where $\mu^{\prime}$ and $\mu^{\prime \prime}$ are distinct but have the same marginals. By the Hann-Banach theorem, there exists a continuous functional $\theta$ of norm 1 such that $\theta$ is identically zero on $\mathcal{S}$. Since the dual space to $\mathcal{L}^{1}(\mu)$ is the space $\mathcal{L}^{\infty}(\mu)$ of essentially bounded functions, the functional $\theta$ can be identified with a nonzero function $\theta\left(x_{1}, \ldots, x_{n}\right)$ bounded by 1 in absolute value. The condition of vanishing on $\mathcal{S}$ reads as

$$
\int \sum_{i \in N} g_{i}\left(x_{i}\right) \cdot \theta\left(x_{1}, \ldots, x_{n}\right) \mathrm{d} \mu=0, \quad \forall g_{i}=g_{i}\left(x_{i}\right) \in L^{1}(\mu), i \in N .
$$

We see that the measure $\theta \mathrm{d} \mu$ is nonzero but has zero marginals! Define $\mu^{\prime}$ and $\mu^{\prime \prime}$ as $\mathrm{d} \mu^{\prime}=(1-\theta) \mathrm{d} \mu$ and $\mathrm{d} \mu^{\prime \prime}=(1+\theta) \mathrm{d} \mu$, respectively. By construction, $\mu$ is the average of $\mu^{\prime}$ and $\mu^{\prime \prime}, \mu^{\prime}$ and $\mu^{\prime \prime}$ are distinct, and all measures $\mu, \mu^{\prime}$, and $\mu^{\prime \prime}$ have the same marginals. Thus, $P$ is also represented as the average of $\mu^{\prime}+\lambda^{\perp}$ and $\mu^{\prime \prime}+\lambda^{\perp}$; that is, $P$ is not an extreme point. A contradiction results. This contradiction completes the proof (under the assumption that $\mathcal{S} \neq \mathcal{L}^{1}(\mu)$ ).

Now we check that $\mathcal{S} \neq \mathcal{L}^{1}(\mu)$. Our goal is to construct a function $h \in \mathcal{L}^{1}(\mu)$ such that for any $g\left(x_{1}, \ldots, x_{n}\right)=\sum_{i \in N} g_{i}\left(x_{i}\right) \in \mathcal{L}^{1}(\mu)$, the $\mathcal{L}^{1}$ distance $\int|h-g| \mathrm{d} \mu$ is bounded below by some positive constant independent of $g$.

Recall that $\mathrm{d} \mu=f \mathrm{~d} \lambda$. Fix $\delta>0$ such that the set $A_{\delta}=\left\{x \in[0,1]^{N}: f(x) \geq \delta\right\}$ has a nonzero Lebesgue measure. Fix another small constant $\varepsilon>0$; by the Lebesgue density theorem applied to $A_{\delta}$, there exist a point $x^{0} \in(0,1)^{N}$ and a number $a>0$ such that for the cube $C=\prod_{i \in N}\left[x_{i}^{0}-a, x_{i}^{0}+a\right) \subset[0,1]^{N}$ of size $2 a$ centered at $x^{0}$, the following inequality holds: $\lambda\left(C \backslash A_{\delta}\right) / \lambda(C) \leq \varepsilon$.

Define $h(x)=1$ if $x_{i} \geq x_{i}^{0}$ for all $i \in N$ and $h(x)=0$ otherwise.

Cut the cube $C$ into $2^{n}$ small cubes indexed by subsets of $N$ : for $M \subset N$, the cube $C_{M}$ is given by $\prod_{i \in N \backslash M}\left[x_{i}^{0}-a, x_{i}^{0}\right) \times \prod_{i \in M}\left[x_{i}^{0}, x_{i}^{0}+a\right)$. No function $g(x)=$ $\sum_{i \in N} g_{i}\left(x_{i}\right)$ can approximate $h$ well on all the small cubes at the same time. The

\footnotetext{
${ }^{24}$ We refer here to separability in the economic sense.
} 
intuition is the following: since $h$ is zero on all the cubes except $C_{N}$, then $g$ must be close to zero on these cubes; however, values on these cubes determine values of $g$ on $C_{N}$ by

$$
\begin{gathered}
g(x)=\frac{1}{n-1}\left(\left(\sum_{i \in N} g\left(x_{1}, \ldots, x_{i-1}, x_{i}-a, x_{i+1}, \ldots, x_{n}\right)\right)\right. \\
\left.-g\left(x_{1}-a, x_{2}-a, \ldots, x_{n}-a\right)\right),
\end{gathered}
$$

and therefore, $g$ is close to zero on $C_{N}$ and cannot approximate $h$ well.

To formalize this intuition, we assume that $\int_{C_{M}}|h-g| \mathrm{d} \mu$ is less than some $\alpha \cdot \lambda\left(C_{M}\right)$ for any $M \subset N$ and show that this constant $\alpha$ cannot be too small. For $M \neq N$, we get $\int_{C_{M}}|g| \cdot f \mathrm{~d} \lambda \leq \alpha \lambda\left(C_{M}\right)$. Applying the Markov inequality on the set $C_{M} \cap A_{\delta}$ and taking into account that this set is big enough (by the construction of the original cube, $\left.\lambda\left(C_{M} \backslash A_{\delta}\right) / \lambda\left(C_{M}\right) \leq 2^{n} \varepsilon\right)$, we obtain the existence of a set $B_{M} \subset C_{M}$ such that $|g(x)| \leq \alpha^{1 / 2} / \delta$ on $B_{M}$ and $\lambda\left(C_{M} \backslash B_{M}\right) / \lambda\left(C_{M}\right) \leq 2^{n} \varepsilon+\alpha^{1 / 2}$.

Consider a subset $B^{*}$ of $C_{N}$ such that, whenever $x \in B^{*}$, all the arguments of the right-hand side in equation (E2) belong to respective subsets $B_{M}$, that is, $B^{*}=\bigcap_{i \in N}\left(B_{N \backslash\{i\}}+a \cdot e_{i}\right) \cap\left(B_{\varnothing}+a \cdot \sum_{i \in N} e_{i}\right)$, where sets $B_{M}$ are translated by the elements of the standard basis $\left(e_{i}\right)_{i \in N}$. The union bound implies that the set $B^{*}$ is dense enough in $C_{N}: \lambda\left(C_{N} \backslash B^{*}\right) / \lambda\left(C_{N}\right) \leq(n+1)\left(2^{n} \varepsilon+\alpha^{1 / 2}\right)$. By formula (E2), the absolute value of $g$ is bounded by $[n /(n-1)]\left(\alpha^{1 / 2} / \delta\right)$ on $B^{*}$. We get the following chain of inequalities:

$$
\begin{aligned}
\alpha & \geq \frac{1}{\lambda\left(C_{N}\right)} \int_{C_{N}}|h-g| \mathrm{d} \mu \\
& \geq \frac{1}{\lambda\left(C_{N}\right)} \int_{B^{*} \cap A_{\delta}}|h-g| \cdot f \mathrm{~d} \lambda \\
& \geq\left(1-\frac{n}{n-1} \frac{\sqrt{\alpha}}{\delta}\right) \cdot \delta \cdot \frac{\lambda\left(B^{*} \cap A_{\delta}\right)}{\lambda\left(C_{N}\right)} \\
& \geq\left(\delta-\frac{n}{n-1} \sqrt{\alpha}\right)\left[1-(n+1)\left(2^{n} \varepsilon+\sqrt{\alpha}\right)-2^{n} \varepsilon\right] .
\end{aligned}
$$

Denote by $\alpha^{*}=\alpha^{*}(\delta, n, \varepsilon)$ the minimal value of $\alpha \geq 0$ satisfying the inequality created by the head and the tail of (E3). The constant $\varepsilon$ is a free parameter in the construction. Selecting it to be small enough (namely, $(n+2) 2^{n} \varepsilon<1$ ), we ensure that $\alpha^{*}>0$.

For any $g=\sum_{i \in N} g_{i}\left(x_{i}\right)$,

$$
\int_{[0,1]^{]^{N}}}|h-g| \mathrm{d} \mu \geq \max _{M \subset N} \int_{C_{M}}|h-g| \mathrm{d} \mu \geq \alpha^{*}(\delta, n, \varepsilon) \cdot 2^{-n} \lambda(C)>0 .
$$

Since the constant on the right-hand side is independent of $g$ and positive, we see that $h$ cannot be approximated by separable functions. Thus, $h$ does not belong to $\mathcal{S}$, and $\mathcal{S} \neq \mathcal{L}^{1}$. QED 
Appendix F

\section{Independent Beliefs Induced by Gaussian Signals}

Let $\phi$ be the density of the standard Gaussian random variable: $\phi(t)=$ $\left[1 /(2 \pi)^{1 / 2}\right] e^{-\left(t^{2} / 2\right)}$.

For prior $p=1 / 2$, consider an agent who gets a signal $s \in \mathbb{R}$ distributed according to the Gaussian distribution with variance 1 and mean equal to $d$ for the state $\omega=\mathrm{h}$, and equal to $-d$ for the state $\omega=\ell$, so that the conditional distributions have the densities $\phi(s-d)$ and $\phi(s+d)$, respectively. The density $f(s)$ of the unconditional distribution is

$$
f(s)=\frac{1}{2} \phi(s-d)+\frac{1}{2} \phi(s+d)=\frac{1}{2 \sqrt{2 \pi}} e^{-d^{2} / 2} e^{-s^{2} / 2}\left(e^{d s}+e^{-d s}\right) .
$$

For the sake of definiteness, we assume $d>0$.

Denote by $\nu$ the induced distribution of posteriors. By Bayes's law, the posterior $x(s)$ upon receiving the signal $s$ is equal to

$$
x(s)=\frac{\phi(s-d)}{(1 / 2) \phi(s-d)+(1 / 2) \phi(s+d)} \cdot \frac{1}{2}=\frac{e^{d s}}{e^{d s}+e^{-d s}}
$$

and

$$
\nu([0, t])=\int_{-\infty}^{x^{-1}(t)} f(s) \mathrm{d} s .
$$

We are interested in the question of when $\nu \times \nu$ is 1/2-feasible. By proposition 2, it is feasible if and only if it is a mean-preserving contraction of the uniform distribution. The next lemma provides a simple sufficient condition for this property.

Lemma 7 . Let $\mu \in \Delta([0,1])$ be a nonatomic distribution, symmetric around $1 / 2$, with cumulative distribution function $F(a)=\mu([0, a])$. Assume that there is at most one point $1 / 2<a<1$ such that $F(a)=a$ and that $F(x)-x>0$ for all $x$ close enough to 1 but not equal to 1 . Then, the uniform distribution is a mean-preserving spread of $\mu$ if and only if

$$
\frac{1}{8} \geq \int_{1 / 2}^{1}\left(x-\frac{1}{2}\right) \mathrm{d} \mu(x) .
$$

Proof. Necessity follows directly from the convex order definition of meanpreserving spreads. Indeed, the condition for being a mean-preserving contraction of the uniform distribution is equivalent to

$$
H(y)=\int_{0}^{y}(F(x)-x) \mathrm{d} x \leq 0 \quad \forall y \in[0,1] .
$$

Integration by parts implies that for $a=1 / 2$ this condition becomes exactly condition (F2).

To prove sufficiency, we must check the inequality (F3) for all $y \in[0,1]$. Symmetry of $\mu$ implies $H(y)=H(1-y)$, and thus we can focus on the right-hand subinterval $y \in[1 / 2,1]$. It is enough to check that $\max _{y \in 1 / 2,1]} H(y) \leq 0$. We claim that the maximum is attained at one of the end points. Indeed, if there is an internal 
maximum $a \in(1 / 2,1)$, then the derivative $H^{\prime}(a)=F(a)-a=0$. On the whole interval $(a, 1)$, the derivative $H^{\prime}(y)$ has a constant sign, since it is continuous and $y=a$ is the unique zero in $(1 / 2,1)$. By assumption, $F(x)-x>0$ for $x$ close to 1 , and hence the derivative is positive on $(a, 1)$ and $H$ is increasing on this interval. Therefore, the point $a$ cannot be a maximum of $H$ on $[1 / 2,1]$. Thus, condition (F3) holds if and only if it holds on the end points $y=1 / 2$ and $y=1$. For $y=1 / 2$, this inequality coincides with condition (F2), and for $y=1$ it is trivial. QED

Below, we check that $\nu$, as induced by Gaussian signals, satisfies the assumptions of lemma 7 and that therefore $\nu \times \nu$ is $1 / 2$-feasible if and only if $\int_{1 / 2}^{1}(x-$ $1 / 2) \mathrm{d} \nu \leq 1 / 8$. This is equivalent to $\int_{1 / 2}^{1} x \mathrm{~d} \nu \leq 3 / 8$.

We can make this condition more explicit by rewriting its left-hand side as

$$
\int_{1 / 2}^{1} x \mathrm{~d} \nu(x)=\int_{0}^{\infty} x(s) \cdot f(s) \mathrm{d} s=\frac{1}{2} \int_{0}^{\infty} \phi(s-d) \mathrm{d} s=\frac{1}{2} \int_{-\infty}^{d} \phi(t) \mathrm{d} t,
$$

where in the last equation we applied the change of variable $s-d=-t$. This results in the following condition of feasibility:

$$
\int_{-\infty}^{\mathrm{d}} \phi(t) \mathrm{d} t \leq \frac{3}{4}
$$

that is, $d$ must be below the $3 / 4$ quantile of the standard normal distribution. ${ }^{25}$

It remains to prove that $\nu$ indeed satisfies the assumptions of lemma 7 . We check that $\nu([0, x])-x$ is positive for $x$ close to 1 . Recall that we can write the belief $x$ as a function $x(s)$ of the signal $s$, using equation (F1). We denote the derivative of $x(s)$ by $x^{\prime}(s)$. Substituting $x=x(s)$, we get

$$
\nu([0, x(s)])-x(s)=1-x(s)-\nu([x(s),+\infty))=\int_{s}^{\infty}\left(x^{\prime}(t)-f(t)\right) \mathrm{d} t .
$$

For large $t$, we have

$$
\begin{aligned}
& f(t)=\frac{1}{2 \sqrt{2 \pi}} e^{-d^{2} / 2} \cdot e^{-t^{2} / 2+d \cdot t}(1+o(1)) \quad \text { and } \\
& x^{\prime}(t)=\frac{2 d}{\left(e^{d \cdot t}+e^{-d \cdot t}\right)^{2}}=2 d \cdot e^{-2 d \cdot t}(1+o(1)) .
\end{aligned}
$$

Therefore, the asymptotic behavior of the integrand is dictated by $x^{\prime}(t)$ :

$$
x^{\prime}(t)-f(t)=x^{\prime}(t)(1+o(1)) .
$$

The integrand is positive for $t$ large enough. This implies the desired positivity of $\nu([0, x(s)])-x(s)$ for large values of $s$.

Now we check that there is at most one point $a \in(1 / 2,1)$ such that $F(a)=a$ or, equivalently, that there is at most one point $s \in(0, \infty)$ such that $\int_{-\infty}^{s} f(t) \mathrm{d} t-$ $x(s)=0$. Denote $G(s)=\int_{-\infty}^{s} f(t) \mathrm{d} t-x(s)$. The function $G$ is smooth, and

${ }_{25}$ Note that in this computation, we do not use the explicit formula for $\phi$. In particular, one gets the same condition of feasibility for any absolutely continuous distribution of signals on $\mathbb{R}$, if the induced distribution of posteriors $\nu$ satisfies the assumptions of lemma 7 . 
$G(0)=\lim _{s \rightarrow+\infty} G(s)=0$. If $G$ has $k$ zeros in $(0, \infty)$, then it also has at least $k+1$ extrema (minima or maxima) in this interval and hence at least $k+1$ critical points (zeros of the derivative $G$ ). We show here that there are no more than two critical points and thus that $G$ has at most one zero. The equation for critical points takes the following form:

$$
G^{\prime}(s)=0 \Leftrightarrow \frac{1}{2 \sqrt{2 \pi}} e^{-d^{2} / 2} e^{-s^{2} / 2}\left(e^{d \cdot s}+e^{-d \cdot s}\right)-\frac{2 d}{\left(e^{d \cdot s}+e^{-d \cdot s}\right)^{2}}=0
$$

and can be rewritten as

$$
e^{-s^{2} / 2}\left(e^{d \cdot s}+e^{-d \cdot s}\right)^{3}=4 d \sqrt{2 \pi} e^{d^{2} / 2} .
$$

Denote the left-hand side by $H(s)$. The graph of $H$ for $s \geq 0$ can intersect any given level at most twice, since $H^{\prime}$ has at most one zero in $(0,+\infty)$. Indeed, $H^{\prime}(s)=$ $(s-3 d \cdot \tanh (d q)) \cdot H(q)$, and the equation $q-3 d \cdot \tanh (d q)=0$ has at most one positive solution, by concavity of the hyperbolic tangent on $[0,+\infty)$. Therefore, $G$ has at most two critical points and thus at most one zero in $(0, \infty)$, which completes the argument and justifies the application of lemma 7 to $\nu$.

\section{References}

Arieli, Itai, and Yakov Babichenko. 2019. "Private Bayesian Persuasion.” J. Econ. Theory 182:185-217.

Arieli, Itai, Yakov Babichenko, and Rann Smorodinsky. 2020. "Identifiable Information Structures." Games and Econ. Behavior 120:16-27.

Aumann, Robert J. 1976. "Agreeing to Disagree." Ann. Statis. 4 (6): 1236-39.

Aumann, Robert J., and Michael Maschler. 1995. Repeated Games with Incomplete Information. In collaboration with Richard E. Stearns. Cambridge, MA: MIT Press.

Bergemann, Dirk, Benjamin Brooks, and Stephen Morris. 2017. "First-Price Auctions with General Information Structures: Implications for Bidding and Revenue." Econometrica 85 (1): 107-43.

Bergemann, Dirk, Tibor Heumann, and Stephen Morris. 2021. "Information, Market Power and Price Volatility." RAND J. Econ. 52 (1): 125-50.

Bergemann, Dirk, and Stephen Morris. 2019. "Information Design: A Unified Perspective." J. Econ. Literature 57 (1): 44-95.

Blackwell, David. 1951. "Comparison of Experiments." In Proceedings of the Second Berkeley Symposium on Mathematical Statistics and Probability, edited by Jerzy Neyman, 93-102. Berkeley: Univ. California Press. 265-72.

Börgers, Tilman, and Jiangtao Li. 2019. "Strategically Simple Mechanisms." Econometrica 87 (6): 2003-35.

Brooks, Benjamin, and Songzi Du. 2019. "Optimal Auction Design with Common Values: An Informationally-Robust Approach.” http://dx.doi.org/10 $.2139 /$ ssrn.3137227.

Brooks, Benjamin, Alexander P. Frankel, and Emir Kamenica. 2019. "Information Hierarchies.” http://dx.doi.org/10.2139/ssrn.3448870.

Burdzy, Krzysztof, and Soumik Pal. 2019. "Contradictory Predictions." https:// arxiv.org/abs/1912.00126. 
Burdzy, Krzysztof, and Jim Pitman. 2020. "Bounds on the Probability of Radically Different Opinions." Electronic Communications Probability 25:14. http://dx.doi .org/10.1214/20-ECP296.

Cichomski, Stanisław. 2020. "Maximal Spread of Coherent Distributions: A Geometric and Combinatorial Perspective." https://arxiv.org/abs/2007.08022.

Dawid, A., M. H. DeGroot, J. Mortera, et al. 1995. "Coherent Combination of Experts' Opinions." Test 4 (2): 263-313.

Dubins, Lester E., and Jim Pitman. 1980. "A Maximal Inequality for Skew Fields." Zeitschrift Wahrscheinlichkeitstheorie und verwandte Gebiete 52 (3): 219-27.

Ely, Jeffrey C. 2017. "Beeps.” A.E.R. 107 (1): 31-53.

Feinberg, Yossi. 2000. "Characterizing Common Priors in the Form of Posteriors." J. Econ. Theory 91 (2): 127-79.

Gale, David. 1957. "A Theorem on Flows in Networks.” Pacific J. Math. 7 (2): 1073-82.

Gershkov, Alex, Jacob K. Goeree, Alexey Kushnir, Benny Moldovanu, and Xianwen Shi. 2013. "On the Equivalence of Bayesian and Dominant Strategy Implementation." Econometrica 81 (1): 197-220.

Gershkov, Alex, Benny Moldovanu, and Philipp Strack. 2018. "A Theory of Auctions with Endogenous Valuations." http:/ /dx.doi.org/10.2139/ssrn.3097217.

Gutmann, Sam, J. H. B. Kemperman, J. A. Reeds, and L. A. Shepp. 1991. "Existence of Probability Measures with Given Marginals." Ann. Probability 19 (4): 1781-97.

Hansel, Georges, and Jean-Pierre Troallic. 1986. "Sur le problème des marges." Probability Theory and Related Fields 71 (3): 357-66.

Hart, Sergiu, and Philip J. Reny. 2015. "Implementation of Reduced Form Mechanisms: A Simple Approach and a New Characterization." Econ. Theory Bull. 3 (1): $1-8$.

Kamenica, Emir, and Matthew Gentzkow. 2011. "Bayesian Persuasion." A.E.R. 101 (6): 2590-615.

Kellerer, Hans G. 1961. "Funktionen auf Produkträumen mit vorgegebenen Marginal-Funktionen." Mathematische Annalen 144 (4): 323-44.

Levy, Gilat, Inés Moreno de Barreda, and Ronny Razin. 2020. "Persuasion with Correlation Neglect." Working paper.

Lindenstrauss, Joram. 1965. "A Remark on Extreme Doubly Stochastic Measures." American Math. Monthly 72 (4): 379-82.

Lorentz, G. G. 1949. "A Problem of Plane Measure." American J. Math. 71 (2): 417-26.

Mathevet, Laurent, Jacopo Perego, and Ina Taneva. 2020. "On Information Design in Games." J.P.E. 128 (4): 1370-404.

Milgrom, Paul, and Nancy Stokey. 1982. "Information, Trade and Common Knowledge." J. Econ. Theory 26 (1): 17-27.

Monderer, Dov, and Dov Samet. 1989. "Approximating Common Knowledge with Common Beliefs." Games and Econ. Behavior 1 (2): 170-90.

Morris, Stephen E. 1991. "The Role of Beliefs in Economic Theory." PhD diss., Yale Univ. https://economics.mit.edu/files/19990.

_ 2020. "No Trade and Feasible Joint Posterior Beliefs." Working paper. https://economics.mit.edu/files/20126.

Neeman, Zvika. 1996. "Approximating Agreeing to Disagree Results with Common p-Beliefs." Games and Econ. Behavior 12 (1): 162-64.

Neumann, Michael M. 1985. "The Theorem of Gale for Infinite Networks and Applications." In Infinite Programming, edited by Edward J. Anderson and Andrew B. Philpott, 154-71. Berlin: Springer. 
Rubinstein, Ariel. 1989. "The Electronic Mail Game: Strategic Behavior under 'Almost Common Knowledge'." A.E.R. 79 (3): 385-91.

Ryser, H. J. 1957. "Combinatorial Properties of Matrices of Zeros and Ones." Canadian J. Math. 9:371-77.

Samet, Dov. 1998. "Common Priors and Separation of Convex Sets." Games and Econ. Behavior 24:172-74.

Shortt, R. M. 1986. "The Singularity of Extremal Measures." Real Analysis Exchange 12 (1): 205-15.

Strassen, V. 1965. "The Existence of Probability Measures with Given Marginals." Ann. Math. Statis. 36 (2): 423-39.

Taneva, Ina. 2019. "Information Design." American Econ. J.: Microeconomics 11 (4): $151-85$.

Weinstein, Jonathan, and Muhamet Yildiz. 2007. "Impact of Higher-Order Uncertainty." Games and Econ. Behavior 60 (1): 200-212.

Ziegler, Gabriel. 2020. “Adversarial Bilateral Information Design.” Working paper. 